\title{
Automated Image Mapping and Quantification of Microstructure Heterogeneity in Additive Manufactured Ti6Al4V
}

DOI:

10.1016/j.matchar.2018.10.027

\section{Document Version}

Accepted author manuscript

Link to publication record in Manchester Research Explorer

Citation for published version (APA):

Zhao, H., Ho, A., Davis, A., Antonysamy, A. A., \& Prangnell, P. (2019). Automated Image Mapping and Quantification of Microstructure Heterogeneity in Additive Manufactured Ti6Al4V. Materials Characterization, 147, 131-145. https://doi.org/10.1016/j.matchar.2018.10.027

\section{Published in:}

Materials Characterization

\section{Citing this paper}

Please note that where the full-text provided on Manchester Research Explorer is the Author Accepted Manuscript or Proof version this may differ from the final Published version. If citing, it is advised that you check and use the publisher's definitive version.

\section{General rights}

Copyright and moral rights for the publications made accessible in the Research Explorer are retained by the authors and/or other copyright owners and it is a condition of accessing publications that users recognise and abide by the legal requirements associated with these rights.

\section{Takedown policy}

If you believe that this document breaches copyright please refer to the University of Manchester's Takedown Procedures [http://man.ac.uk/04Y6Bo] or contact uml.scholarlycommunications@manchester.ac.uk providing relevant details, so we can investigate your claim.

\section{OPEN ACCESS}




\title{
Automated Image Mapping and Quantification of Microstructure Heterogeneity in Additive Manufactured Ti6Al4V
}

\author{
Hao Zhao a, b, Alistair Ho ${ }^{\mathrm{a}}$, Alec Davis ${ }^{\mathrm{a}}$, Alphons Antonysamy ${ }^{\mathrm{c}}$, Phillip Prangnell ${ }^{\mathrm{a}}$ \\ ${ }^{\text {a }}$ School of Materials, University of Manchester, Manchester M13 9PL, UK \\ b 3DPTek Co., Ltd., Beijing, China \\ ${ }^{\mathrm{c}}$ Additive Manufacturing Centre, GKN Aerospace, Bristol BS34 7QQ, UK
}

\begin{abstract}
In Additive Manufacturing AM, each volume of material experiences a complex thermal history due to both short-range effects, from the repeated overlap of the thermal field from each heat source pass, and long-range variation in the thermal boundary conditions, related to the part geometry and build height. With an $\alpha+\beta$ alloy, like Ti64, this can lead to significant local variation in the transformation microstructure, which can contribute to heterogeneity in the mechanical properties of a component. In order to better understand the transformation microstructure variability in AM parts, an automated microstructure analysis tool has been developed, and tested against independently measured data, that can accurately map the inter-lamellar spacing of the $\alpha$ phase and spheroidicity of the $\beta$ phase, at both high resolution and over large distances. The approach used was based on automated batch image analysis of thousands of image tiles obtained using a mapping function in a high-resolution SEM with a scanning stage. Within a practical operating range of drift in the microscope parameters (e.g. working distance, detector contrast) the errors in the measurements were found to be minimal $(<3 \%)$. Results are discussed from applying the method to two example case studies from different ends of the AM spectrum; selective Electron Beam Melting (EBM) and WireArc Additive Manufactured (WAAM). In the former case this revealed considerable drift in the microstructure with build height and geometry, but little short-range variation, whereas with the WAAM process more severe short range microstructural gradients associated with HAZ banding were fully quantified.
\end{abstract}




\subsection{Introduction}

Titanium alloys are important structural materials in aerospace and the biomedical sector, owing to their high specific strength and excellent corrosion resistance [1]. Because they are expensive to buy and challenging to machine, titanium alloys are well positioned to benefit from the near-net-shape capability of Additive Manufacturing (AM) [2-4]. The concept of AM is based on dividing a 3D object into consecutive 2D slices and consolidating each part, layer by layer, using a scanned focused heat source [2-4]. Additive processes are advantageous over conventional subtractive manufacture (e.g. forging and machining) due to their higher material utilisation, greater design freedom they provide, and the new metallurgical possibilities they can facilitate for tailoring properties through varying the conditions while building components [2-6].

The titanium alloy Ti-6Al-4V (Ti64) is widely used in industry and has been extensively investigated for AM applications (e.g. [2-7]). In AM, each local volume of material can experience a different and complex thermal history, due to short-range effects from the repeated overlap of the thermal field generated by each heat source pass (both horizontally within the deposited layers and vertically between layers) and from long-range drift in the thermal boundary conditions related to the part geometry and build height [6-12]. For example, in powder bed systems the rate of heat loss to the build plate and surrounding powder bed are different and will affect the background build temperature. This can create long-range thermal gradients that can affect the microstructure development during what can be a prolonged exposure while a part is being built $[5,7,11,12-14]$. In contrast, the moving heat source will generate severe local thermal gradients and cause repeated sharp, short, temperature rises and high cooling rates with a diminishing peak temperature, the duration of which depends largely on the scanning speed $[7,9,10,15,16]$. The conditions can vary dramatically depending on the AM process. For example the Electron Beam Melting (EBM) powder bed process uses a small $\left(\sim 100 \mu \mathrm{m}\right.$ diameter) rapidly moving melt pool $\left(\sim 500 \mathrm{~mm} \mathrm{~s}^{-1}\right)$ and a preheated bed maintained at $>700{ }^{\circ} \mathrm{C}$ for several hours (typically $4-12 \mathrm{hrs}$ ) which results in cooling rates in the range $10^{2}-10^{4} \mathrm{~K} \mathrm{~s}^{-1}[7,12]$, compared to Directed Energy high Deposition (DED) rate deposition processes like Wire Arc-plasma Additive Manufacturing (WAAM), which have a large ( $5 \mathrm{~mm})$ diameter slower moving melt pool $\left(\sim 5 \mathrm{~mm} \mathrm{~s}^{-1}\right)$ and usually operate with a cold substrate and have cooling rates in the range $10^{1}-10^{2} \mathrm{~K} \mathrm{~s}^{-1}[10,16,17]$.

The complex thermal history in AM with Ti64 is known to influence a variety of important microstructural features that affect a component's properties and that can become heterogeneously distributed within a part, including; the prior $\beta$ grain size [11,12], grain boundary $\alpha$ layer $[12,18,19], \alpha$ colony size $[12,19], \alpha$ plate spacing [12-14,20], and phase morphology $[13,18]$. In most AM Ti64 parts owing to the high cooling rate the final bulk microstructure is dominated by a basket weave Widmanstätten $\alpha$ microstructure, which is comprised of fine interwoven $\alpha$ lamellae with a small fraction of retained $\beta$ [7,8,12-14,18-20]. However, in high cooling rate powder bed processes like Selective Laser Melting $(\mathrm{SLM})\left(\dot{T}=10^{3}-10^{4} \mathrm{~K} \mathrm{~s}^{-1}\right)$, the as-built microstructure is 
generally martensitic, but this is normally decomposed during subsequent heat treatment to form a similar a lamellar dominated microstructure [5,6], whereas in DED processes, such as WAAM, with lower cooling rates the Widmanstätten $\alpha$ microstructure forms directly on cooling after deposition $\left(\dot{T}=10^{1}-10^{2} \mathrm{~K} \mathrm{~s}^{-1}\right)[5,10,16,20,21]$. In EBM of Ti64 martensite has also been observed in the last deposited layers and the transformation microstructure may therefore form first as martensite and decompose in-process to a fine $\alpha-\beta$ morphology owing to the high background build temperature $\left(>700{ }^{\circ} \mathrm{C}\right)[12,18,22]$. In the context of diffusion controlled $\alpha$ transformation microstructures, the $\alpha$ 'colony size' is widely cited as one of the most important microstructure parameters to influence the mechanical properties as it determines the effective slip line length [1,19,23]. However, this terminology strictly applies to wrought products that contain colonies of single $\alpha$ variants that grow in from grain boundaries at much lower cooling rates. Under the high cooling rate conditions in AM, grain boundary colonies are much more restricted to small volumes of material owing to the large $\beta$ grain and size competition with the nucleation of $\alpha$ within the larger primary $\beta$ grains $[1,24]$. This typically occurs with several variants ( $\alpha$ orientations) nucleating within a given volume of material, to produce the classic basket weave microstructure [25]. As such, different sets of variants can nucleate in different parts of a grain leading to impingement of basket weave texture 'colonies' with more diffuse boundaries than grain boundary $\alpha$ colonies. Such microstructures will also typically contain a continuous layer of thin retained $\beta$ of the same orientation as the parent grain between the high aspect ratio $\alpha$ laths $[1,18,25]$. However, repeated thermal cycling to high temperatures where the $\alpha$ phase will start to dissolve can cause significant coarsening and high temperature exposure can also spheroidize the $\beta$ phase [23,26]. During in process, or ex, -situ tempering of martensitic microstructures the supersaturated $\alpha^{\prime}$ phase will decompose by precipitating $\beta$ at the $\alpha^{\prime}$ plate boundaries [1,6,27]. This precipitated form of $\beta$ phase can also become non-continuous and spheroidize during prolonged tempering, due to the tendency to lower the surface energy of the $\alpha-\beta$ interface.

In high deposition rate AM processes like WAAM, which typically have a large layer height (1-2 $\mathrm{mm}$ ) and a low background build temperature, periodic microstructure banding has been widely reported caused by the Heat Affected Zone (HAZ) generated from each heat source pass $[19,21,24,26,28,29]$. The development of such HAZ bands can introduce strong local microstructure gradients within components and were first discussed in detail, based on qualitative observations of the local microstructure coarsening behaviour, by Kelly and Kampe (2004) [24,26]. In contrast, in finer scale AM processes like EBM the transformation microstructure is considered to be more homogeneous because of greater thermal field overlap between multiple tracks and, particularly in the case of EBM, where high build temperatures are used [7,12,8]. However, it is still known to be sensitive to the part geometry [7,11], beam scan control algorithms [11,14,30] build temperature [12] and height $[7,13,14]$. For example, the $\alpha$ lamellar spacing can vary significantly along the build direction $[13,14]$, since the local thermal conditions are affected by the position within the part 
relative to the heat flux through the build plate. Scharowsky et al. have also shown that in EBM the lamellar plate spacing increases with the energy volume density of the heat source [30].

The $\alpha$ lamellar spacing and continuity of the $\beta$ phase are thus key characteristic features of the homogeneity of transformation microstructure seen in AM parts and are closely related to the materials local thermal history. The $\alpha$ lamellar spacing is particularly important because of its correlation to the materials yield stress [31,32], and its heterogeneity will also reflect variability in mechanical performance [7,12,14,19,32]. Mapping of such microstructure parameters across components would thus be useful to provide feedback for process optimisation and model validation, and to better understand the mechanical property variation in AM components.

Despite the obvious benefits, such microstructure parameters have been surprisingly rarely quantified because they are very time consuming to measure manually and it is not a straightforward process to automate $[33,34]$. This is because a high magnification is required to resolve the fine microstructure and there is large local variability within Ti transformation microstructures, which when combined with sectioning effects requiring large numbers of images for statistically valid measurements. In addition, with the most suitable imaging method for resolving the fine nature of the microstructure, which is by backscattered imaging in a high-resolution SEM using Z-contrast between the $\alpha$ and $\beta$ phases, reliably distinguishing the phase boundaries is not a trivial process due to variation in the background diffraction contrast and chemistry of the respective phases. For example, with coarser scale microstructures in wrought titanium where implementation of image analysis is less challenging, Chrapon'sk and Szkliniarz (2001) [33] employed pre-processing before measurement that included: smoothing by a mean filter, histogram normalization, thresholding and noise reduction. More recently, Li et al [34], again in Ti coarser microstructures, have achieved good results by first employing pre-processing using pre-median filtering, a two stage top-hat transformation, and binarization by Ostu's method [35].

Until recently the high magnification required to resolve the microstructure in AM parts has made obtaining quantitative microstructure data from large areas prohibitively labour intensive. However, the capability is now widely available for deploying automated mapping software in high resolution SEM's (e.g. FEI MAPS ${ }^{\mathrm{TM}}$ ) to capture large numbers of images at predetermined locations in a sample. Here, we have exploited this method to investigate the potential for using an automated approach to accurately quantify the heterogeneity of the fine transformation microstructure typically reported in AM components. This has involved developing and testing a reliable image analysis procedure that can batch process large sets of image tiles over long distances (e.g. cm's). Results have been obtained for two AM technologies at different ends of the spectrum, using case study samples built by EBM and WAAM processes, and the data that can be obtained is discussed in relation to the part geometry and process conditions. The same methodology could also be applied to conventional wrought products, but here we have focused on the relatively new field of AM, owing to the large interest in quantifying microstructure heterogeneity in AM processes. 


\subsection{Methodology}

\subsection{Test Specimens}

Two sets of test samples were evaluated to explore the potential for using automated microstructure quantification to measure the heterogeneity found in the fine $\alpha-\beta$ transformation microstructures typically seen in AM components. EBM samples were used to investigate the effect of part geometry and position within a build on both the short and long range heterogeneity in a typical powder bed process, while WAAM samples were used to test the viability of measuring the more severe microstructure gradients arising from HAZ banding in high deposition rate AM parts [21].

\section{EBM samples}

Two main samples types were produced using an Arcam A2 machine at GKN's additive manufacturing centre, UK; a tall vertical square section sample, to investigate the effect of build height in the chamber, and a set of prismatic-shaped samples, to systematically test the influence of changing the part cross-section area and orientation in the build plane (see Figure 1). The feed stock used was gas atomized Ti-6Al-4V powder (standard Grade 5 with oxygen less than 0.2 wt. \%) supplied by Arcam. The particle size of the alloy powder ranged from $45-110 \mu \mathrm{m}$. The standard recommended Arcam Ti64 settings were used for all the test specimens, which included scanning themes 4 for contouring and 19 for infill hatching, a constant build layer height of $50 \mu \mathrm{m}$, and a working temperature of $740{ }^{\circ} \mathrm{C}$.

The $15 \mathrm{~mm}$ square by $\sim 280 \mathrm{~mm}$ tall sample (Figure 1(a)) was built vertically to the maximum height of the build chamber and cross sectioned (the $x-y$ plane) at different positions along its length. The build time for this part was very long and took 36 hours. The first set of square-based $(10 \times 10 \mathrm{~mm})$ prism samples (Figure 1(b)) were built inverted with their tip down to investigate the effect of a low thermal contact with the base plate and the rate of increase in layer cross sectional area (designated P1, P2, P3) with build height. The support structures used are still seen attached (e.g. P1). Samples $\mathrm{V} 1$, and $\mathrm{H} 1$, were built using the same dimensions as prism P2, but with different orientations; V1 (vertical) base down and H1 (horizontal) side down. These sets of samples were all sectioned for analysis across their mid plane. The sectioned planes analysed are indicated in Figure 1. The centre hatch region from a component produced on an older Arcam S12 machine, with a $60 \mu \mathrm{m}$ layer height, was also analysed to see if layer-wise banding could be detected in an EBM part.

\section{WAAM sample}

A WAAM sample was provided as a single pass wide, 20 layer high, wall by the Welding Engineering and Laser Processing Centre at Cranfield University. The sample had been produced using a tungsten inert gas (TIG) torch under ambient atmosphere with a local argon shielding device. The average layer height was approximately $1.5 \mathrm{~mm}$ (Figure 2(b)). Full details of the WAAM process used can be found in references $[20,36]$. The sample was cross sectioned near its top and the last HAZ layer band analysed using a continuous strip of images 5 tiles wide. 
(a)
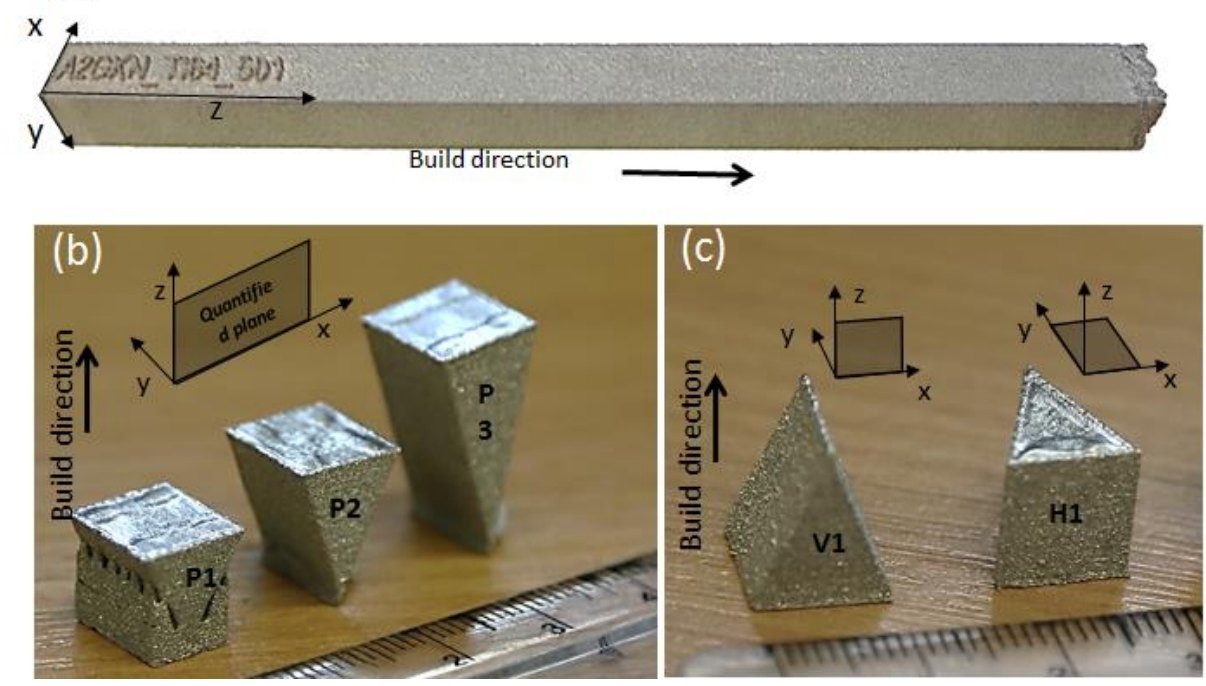

Figure 1. The EBM sample sets studied by automated microstructure mapping; (a) a tall vertical square section sample and prismatic-shaped samples (b) P1-P3 built inverted with different wall angles and supports where required, and (c) the sample shape P2 built in different orientations; vertical V1, horizontal $\mathrm{H} 1$. The standard reference frame used (x, y, z; where $\mathrm{x}-\mathrm{y}$ are the build plane and $\mathrm{z}$ is the build height direction) and planes the samples were sectioned in for analysis are indicated.

Table 1 -EBM and WAAM build parameters

\begin{tabular}{lc|ll} 
Arcam A2 machine & & WAAM & \\
\hline Accelerating voltage & $60 \mathrm{kV}$ & Wire feed speed & $1.6 \mathrm{~m} \mathrm{~min}^{-1}$ \\
Chamber pressure & $4 \times 10^{-3} \mathrm{~Pa}$ & Travel speed & $4.5 \mathrm{~mm} \mathrm{~s}^{-1}$ \\
Contouring theme & 4 & Average current & $110 \mathrm{~A}$ \\
infill hatching theme & 19 & Pulse duration & $0.5 \mathrm{~s}$ \\
build layer height & $50 \mu \mathrm{m}$ & Frequency & $10 \mathrm{~Hz}$ \\
working temperature of & $740^{\circ} \mathrm{C}$ & Gas flow rate & $10 \mathrm{~L} \mathrm{~min}^{-1}$ \\
& & Trailing shield gas flow rate & $20 \mathrm{~L} \mathrm{~min}^{-1}$ \\
& & Torch stand-off & $3.5 \mathrm{~mm}$
\end{tabular}

\subsection{Sample Preparation}

To obtain consistent microstructure data it is important to adopt a standard sample preparation procedure. The Ti-6Al-4V metallography specimens were mounted in conductive resins and first ground using a series of abrasive grinding papers with decreasing grit coarseness: $180-320-600-$ $1200-2500-4000$, each step taking approximately $1 \sim 3$ minutes. A $1 \mu \mathrm{m}$ diameter diamond suspension was then used to polish the sample to a mirror quality. Lastly, polishing with an OPS 
suspension mixed with $5 \%$ concentration hydrogen peroxide $\left(\mathrm{H}_{2} \mathrm{O}_{2}\right)$ was employed to obtain an optimum surface for microscopic observation.

Table 2 The optimised SEM imaging settings and MAPS settings used for the high-resolution imaging and mapping of the EBM and WAAM microstructures.

\begin{tabular}{ll}
\multicolumn{2}{c}{ FEI Magellan SEM - Image settings } \\
\hline Voltage $(\mathrm{kV})$ & 8.0 \\
Current $(\mathrm{nA})$ & 1.6 \\
Field width $(\mu \mathrm{m})$ & $25(\mathrm{WAAM})$ \\
Dwell time $(\mathrm{ms})$ & $50(\mathrm{EBM})$ \\
\end{tabular}
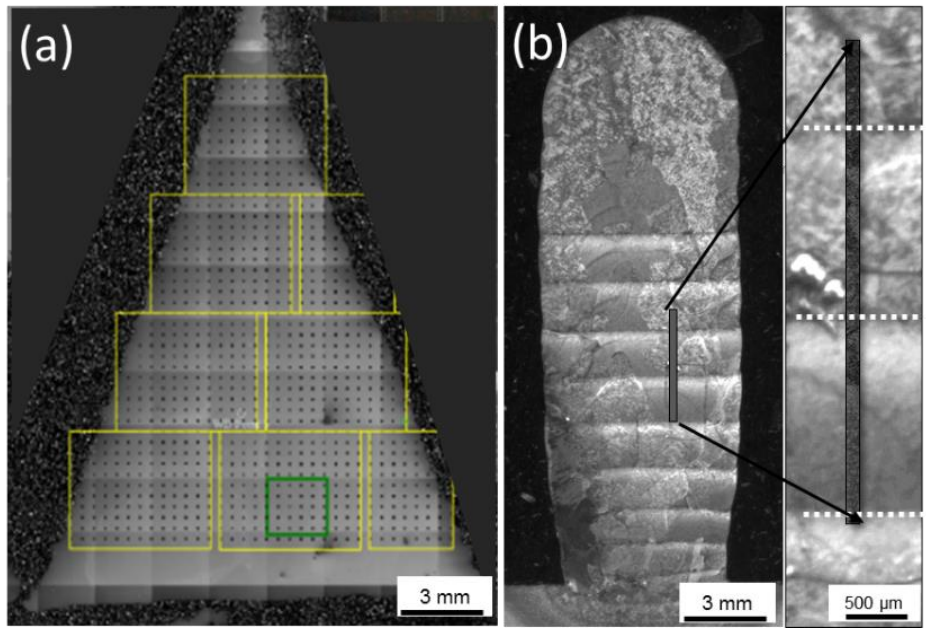

Figure 2 Examples of different automated image mapping strategies adopted for acquiring high resolution data over large areas, shown after mapping with carbon contamination indicating the image capture positions: (a) a large area 2D grid on an EBM prism sample, (b) a continuous 5 tile wide strip from a WAAM wall.

\subsection{Automatic Image Acquisition}

Batch acquisition of SEM images was performed using an FEI Magellan SEM, equipped with a high precision stage and 'MAPS' software, which can automatically take a series of SEM images at predefined areas of interest and then construct a high definition map by automatic stitching. All imaging was performed using the concentric backscattered (CBS) detector. To study short range changes over smaller areas of interest strips of connected image tiles were obtained, whereas to analyse trends over larger distances, images were acquired using a pre-defined grid combined with stage translation, using a regular pitch size set depending on the length scale of interest. Examples of samples after analysis where the imaging positions can be seen from carbon contamination are shown in Figure 2. The field of view (magnification) for each image tile was set in relation to the microstructure scale to be analysed to ensure a sufficient pixel density for reliable quantification $(\sim 10$ pixels per $\beta$ plate width). For the EBM samples the image tile width used was $50 \mu \mathrm{m}$ wide and for the WAAM part, 
which had a finer $\alpha$ lamellar spacing, the image width was selected as $25 \mu \mathrm{m}$. The image conditions for the microscope were optimised to ensure the maximum detector contrast to noise ratio and optimum resolution and are summarised in Table 2.

\subsection{Automated Quantitative image analysis}

To reliably extract microstructure parameters of interest with consistent results and without user intervention from the large sets of images taken with the MAPS software, a GUI (graphic user interface) automated, batch, image processing software was created in Matlab. The key stages involved and the accuracy of the procedure developed are discussed below.

\subsection{Pre-processing}

The contrast in backscattered electron (BSE) imaging of Ti64 AM samples not only represents atomic number differences in the $\alpha$ and $\beta$ phases due to partitioning of $\mathrm{V}$ and $\mathrm{Al}$, but also includes crystallographic diffraction contrast [37]. Captured micrographs thus exhibit inconsistent grayscales (e.g. Figure 3a), which makes reliable image analysis by grayscale thresholding impossible without user intervention. This issue is illustrated in Figure 3(b) where the original image has been directly segmented using Otsu's method [35] without pre-processing. In this example, bright regions of $\alpha$ that diffracted strongly are incorrectly segmented as $\beta$. To minimise this behaviour, an automated preprocessing function was created to optimise the image contrast. This included: (i) applying a lowpass Gaussian filter, which subtracts a Gaussian blurred background from the original image, with a standard deviation $\sigma$ (the extent of blurring) set between the average $\alpha$ plate thickness (in pixels) $L_{\alpha}$ and the average $\beta$ layer thickness $L_{\beta}$, to minimise the unwanted $\alpha$ contrast at a negligible cost of decreased $\alpha-\beta$ phase contrast: (ii) a 2-D median filter with a fixed window size of 3 by 3 pixel was then applied to suppress background noise: (iii) grey-scale contrast optimisation. Noise suppression in step (ii) was found necessary as grayscale thresholding was very sensitive to random noise which could otherwise be incorrectly segmented as $\beta$. However, a drawback of the median filter is that it could disconnect 1-pixel links. To minimise the error from bridging of thin $\beta$ laths the SEM images were taken at a sufficiently high magnification so that the minimum thickness of $90 \%$ of the $\beta$ phase was greater than 2 pixels in width. In the final step (iii) contrast was optimised using the 'imadjust' Matlab function, which remaps the intensity values of an image by expanding the grayscale spectrum, such that $1 \%$ of the original image is saturated at low and high intensities.

The result of each pre-processing step is shown in Figures 3 (c) and (d) and the final pre-processed image in Figure 3(d) can be compared with the original image in Figure 3(a). In Figure 4 a grayscale contrast plot across several typical $\alpha$ laths, shows that after pre-processing the grayscales of the brighter $\alpha$ plates in the original image are effectively flattened to the background level and the contrast adjusted, so that the $\beta$ phase can be more accurately segmented and more reliable edge detection can be applied (Figure 3 (d) and (f)). 

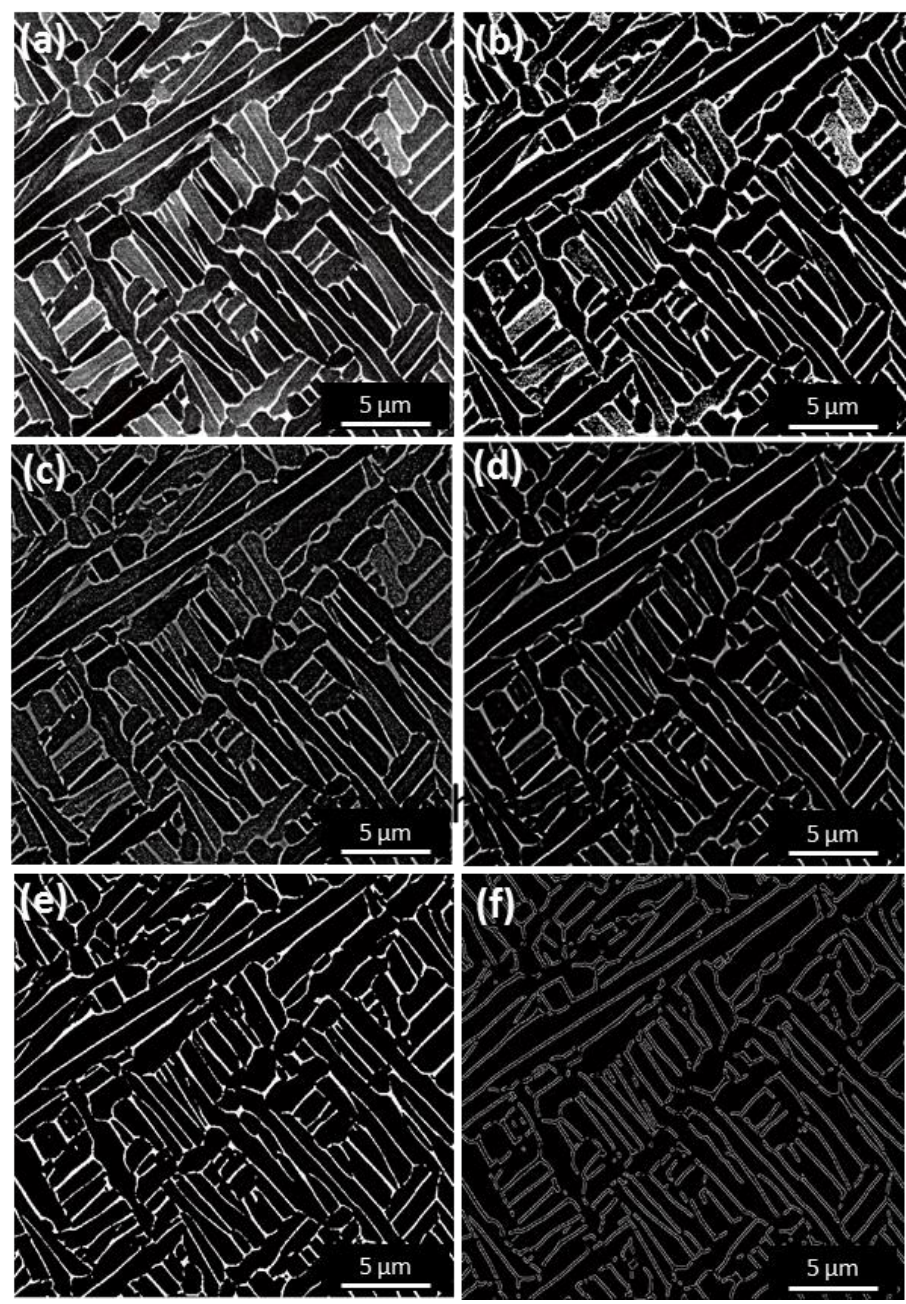

Figure 3. Examples of the effectiveness of image enhancement and post-processing showing: (a) a typical original SEM image of lamellar Ti-6Al-4V microstructure with strong background contrast variation, (b) an Otsu adaptive threshold image without pre-processing, (c) -(d) the pre-processing steps, (c) following application of a low-pass Gaussian filter and (d) after noise reduction and contrast enhancement. The final binarized Ostu [35] adaptive threshold image and edge detected images are shown in (e) and (f) respectively, for comparison with (a).

\subsection{Quantification}

Following pre-processing, to 'quantify' the microstructure features of interest the Ostu adaptive threshold selection method [35] was either directly employed to create a binarized $\alpha-\beta$ image (Figure 3e) and segment the $\beta$ phase, or Canny's edge detection method [38] was used to enable measurement of the $\alpha$ lath 'Inter-Lamellar Spacing' (ILS) using the linear intercept method (Figure 3f).

For the $\alpha$ inter-lamellar spacing measurements Canny's method was first used to define the phase boundaries because it is advantageous over other edge detection methods owing to its higher tolerance to locally varied contrast [39,40]. The method was deployed after first pre-processing, as described above. Each image was then differentiated into a gradient magnitude map (Figure 5a) from 
which edges were detected. In the Canny method edge detection starts from the intensity maxima, such that an edge is grown using a set of decreasing threshold values from low sensitivity (good contrast) regions into high sensitivity (bad contrast) regions (Figure 5). This procedure is followed so that edge detection is less likely to be misled by noise $[38,40]$. To implement this process the maximum and minimum intensity thresholds of the differentiated images were first automatically defined by applying the Ostu adaptive threshold method [35].

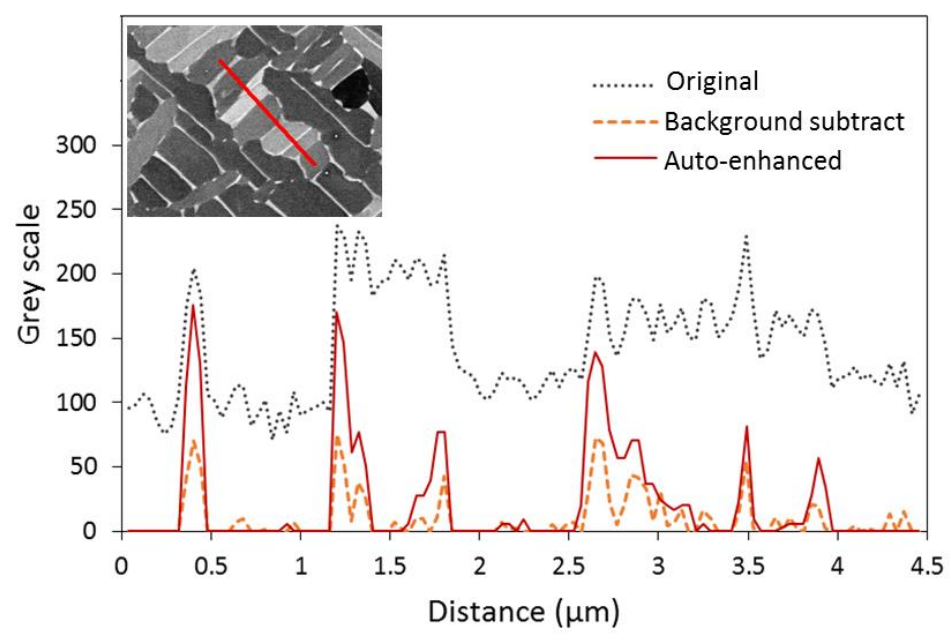

Figure 4. Comparison of the grayscale contrast seen in a typical image, which contains noise and background ( $\alpha$ phase) variation, with that after Gaussian low pass filter background subtraction and auto contrast enhancement.
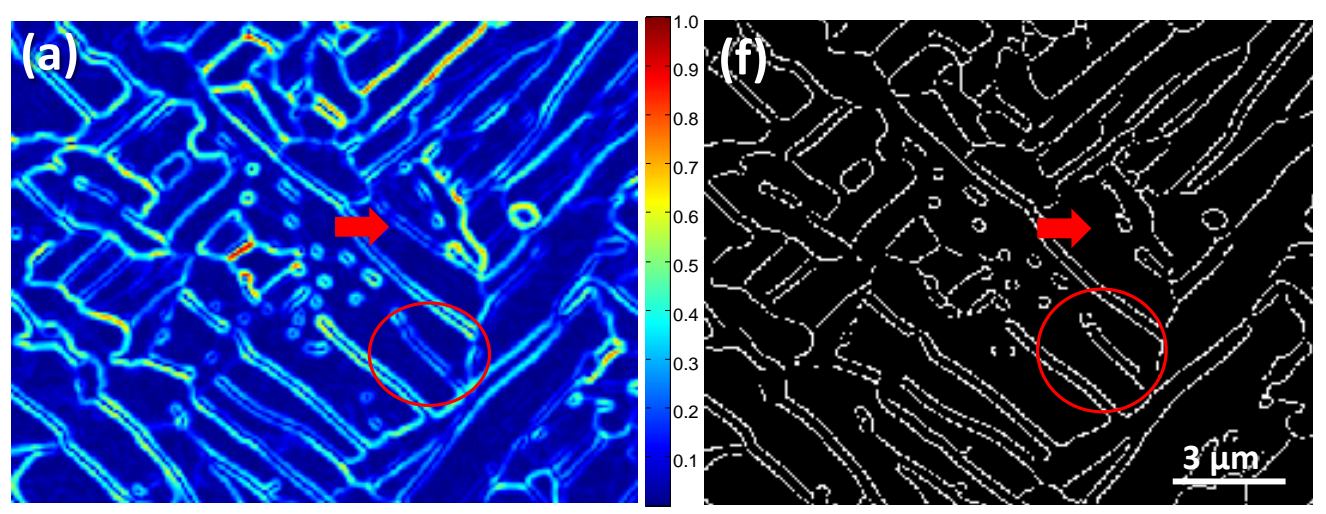

Figure 5. Illustration of the Canny edge detection [38] method; (a) an enlarged differentiated image with (b) the final 'edged' image. By growing 'edges' from high to low contrast areas accurate results are obtained even in regions of low contrast (circled). Occasionally small faint isolated regions can still be missed by the procedure (arrows).

By comparison of the Figure 3(a) and (f) and the enlarged images in Figure 5, it can be seen that overall the agreement between the original image and processed results are very good, with the vast majority of lamellar edges and inter-lamellar $\beta$ being reliably segmented in each case. Despite the pre-processing 
procedure used a small proportion of the image data can still be missed, as exemplified in Figure 5. Nevertheless, this small level of error is greatly outweighed by the ability to rapidly process many images without human bias. Further discussion on the source of potential errors and their magnitude is provided below and in Figs. 6- 8.

The $\alpha$ interlamellar spacing's (ILS) were measured from the final edge detected images, using the standard linear intercept method to obtain the average 2D inter-lamellar spacing from the mean number of intercepts in the horizontal and vertical directions $\bar{\lambda}_{x}, \bar{\lambda}_{y}$, in an image tile width $L_{x}$ and in height $L_{y}$ :

$$
\bar{\lambda}=\frac{1}{\sqrt{\left(\bar{\lambda}_{x} / \bar{L}_{x}\right)^{2}+\left(\bar{\lambda}_{y} / \bar{L}_{y}\right)^{2}}} \quad \text { (Equation 1) }
$$

To save computing time, the interception line pitch was set to 50 pixels for images of $1024 * 884$ resolution, giving 20 horizontal interception lines and 18 vertical lines per image tile.

The binarized images were also used to evaluate the extent to which the $\beta$ phase was affected by thermal exposure after transformation, which can cause it to become partially spheroidized, particularly in the EBM process [18] (see below). This was also an important consideration as spheriodization can affect the identification of the $\alpha$ lath boundaries when using back scattered contrast images. To evaluate such microstructure changes, the segmented $\beta$ regions in the images were registered individually, so that their average aspect ratio, $\beta_{R}$, or circularity, $\beta_{\text {Circ }}$, could be statistically analysed for each image and compared in a batch procedure. Here, circularity is represented as:

$$
\beta_{\text {Circ }}=\frac{4 \pi A}{P^{2}} \quad \text { (Equation 2) }
$$

Where, $\mathrm{A}$, is the area of a segmented $\beta$ particle and, $\mathrm{p}$, is its perimeter.

\subsection{Effect of drift in the imaging conditions}

When collecting many image tiles over large distances with automated stage movement, using automatic focus adjustment and acquisition times of up to 10 hours, drift in the imaging conditions can potentially have a significant influence on the quantification of the BSE images. This can primarily occur due to changes in the working distance, brightness, and contrast levels. To ensure valid measurements it is therefore important to determine the range of imaging conditions that will give reliable results within acceptable limits of practical microscope operation.

In an SEM the working distance (WD) represents the distance from the objective lens to the focal point of the electron beam. It can strongly affect the back diffracted electrons received by the concentric backscattered (CBS) detector, and consequently the signal to noise ratio. Although the sample surfaces were kept as flat and parallel to the focal plane as possible, in practise the working 
distance could vary by the order of $0.1 \mathrm{~mm}$ per $\mathrm{cm}$ of stage translation. When examining long range, component-scale, heterogeneity in AM parts over distances of several cm's the effect of drift in WD is thus an important consideration. To systematically investigate the effect of variation in WD on the microstructure measured parameters and define a stable range that introduces minimal error, a series of micrographs were taken without sample translation while systematically varying the working distance relative to the optimum distance recommended for the Magellan SEM, when using the CBS detector, of $4.0 \mathrm{~mm}$. A distance of $3.7 \mathrm{~mm}$ was selected as the minimum limit, due to safety concerns, giving a range from $3.7 \mathrm{~mm}$ to $4.7 \mathrm{~mm}$. During this test all other SEM settings were kept constant except for the focus which is automatically adjusted.

Examples of a repeated original and processed images obtained as a function of the working distance are shown in Figure 6, where the images have been cropped to a smaller field of view (255 x 235 pixels) to better demonstrate the response of fine-scale features to image processing. The microstructure measurements obtained from each repeated image are summarised in Figure 7(a). It can be seen that the brightness of the BSE image increases with shorter working distances because this provides a greater signal of back-scattered electrons at the CBS detector. However, the optimum contrast is achieved with the manufacturer's recommended WD of $4 \mathrm{~mm}$, where weak $\alpha-\beta$ boundaries (circled in Figure 6) are best retained in the edged image in Figure 6 (b.2), and this leads to the lowest measured ILS value in comparison to the other images (Figure 7a). The contrast to noise level was also maximised with a WD of $4 \mathrm{~mm}$, which after Otsu segmentation gave the maximum continuity of the thin retained $\beta$ layers in Figure 6(c.2). For example, it can be seen that the circled inter- $\alpha$ lath $\beta$ layer appears to degenerate at both higher and shorter working distances. In Figure 7(a) it can also be observed that the measured $\alpha$ ILS results are very consistent within the WD range 3.7 to $4.3 \mathrm{~mm}$, where the minimum values are obtained. The standard deviation in ILS over this range was $0.02 \mu \mathrm{m}$ compared to the mean value of $0.99 \mu \mathrm{m}$, giving a relative maximum error of only about $2 \%$. The mean $\beta$-phase circularity data was also stable between working distances of 3.8 $\mathrm{mm}$ to $4.4 \mathrm{~mm}$, where the relative deviation was only $0.9 \%$. An optimum working distance range for acceptable data was therefore set at $3.8-4.2 \mathrm{~mm}$, which would give an accuracy of better than $98 \%$ for all measurements.

Before batch image acquisition the optimum brightness and contrast levels must be set as in the SEM user interface. However, with large maps taken from region to region, or from specimen to specimen, the imaging conditions inevitably drifts. To compensate for such variations automatic adjustments can be made, but it is still important to determine if drift in the brightness/contrast during acquiring an image map can have a systematic impact on microstructure quantification. An experiment was therefore conducted where the contrast levels were systematically varied using the FEI Magellan SEM settings from 85-98.9. Of these values, 88.1 was the level recommended by the built-in automatic adjustment function in the SEM. While changing the contrast the brightness levels was adjusted to compensate, so as to avoid saturation or too low a signal on the detector, by keeping the 
maximum grey scale close to the detector saturation limit. All other imaging conditions were left unchanged. As before (e.g. Figure 6), the image series obtained was quantified and the resultant data plotted against the microscope contrast setting. It can be seen from Figure 7(b) that as the contrast increases the ILS level is initially quite stable, with only a small deviation of $0.9 \%$, but then it dramatically falls at contrast settings above 91 because of the increasing noise levels. This creates a rising incidence of unwanted features such as internal $\alpha$ plate contrast being mis-segmented as phase boundaries. On the other hand, the $\beta$ circularity was found to be stable between contrast settings of 87 95, which gave a smaller fluctuation of only $0.5 \%$. However, this parameter decreased at high and low contrast settings, respectively, due to a loss of data from thin high aspect-ratio $\beta$ particles at low contrast levels and the greater noise producing rougher phase boundaries at high contrast levels, which exaggerated the $\beta$ phase perimeter.

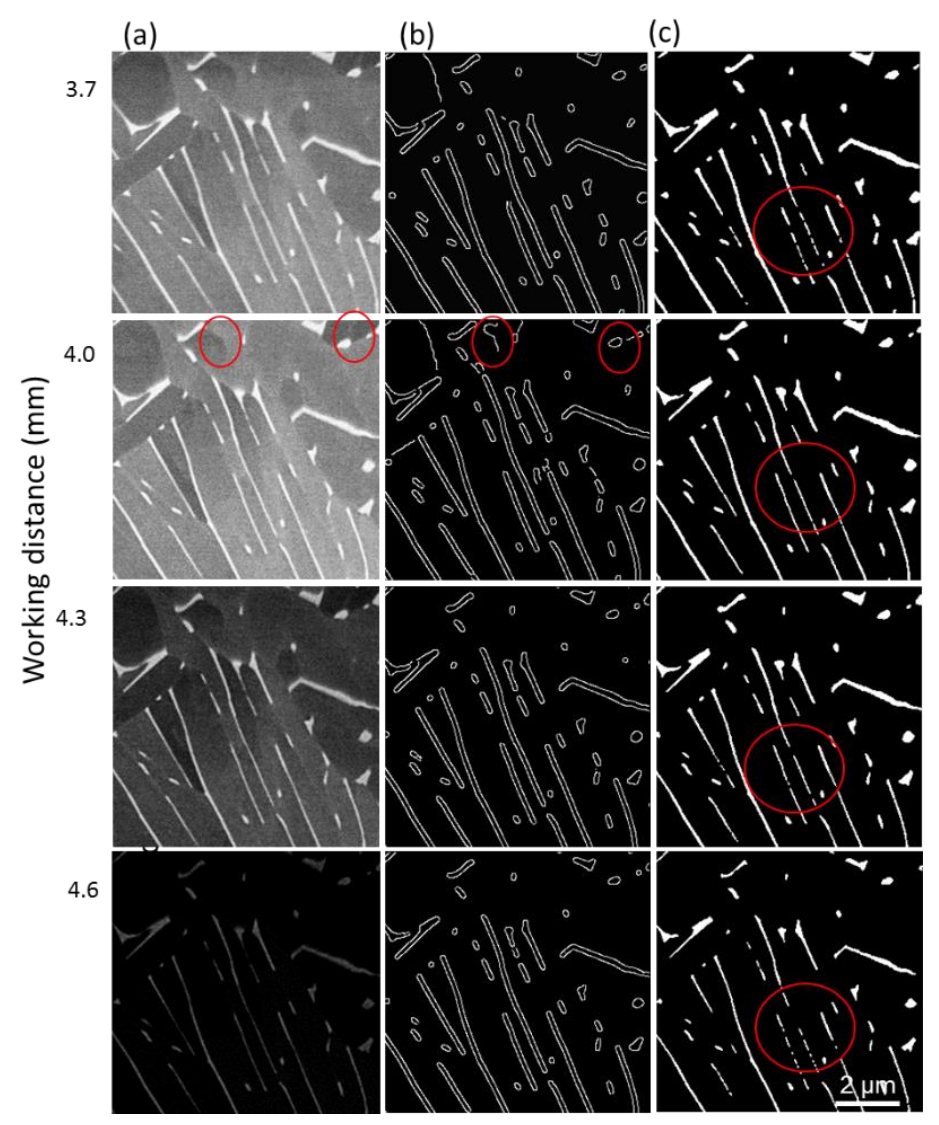

Figure 6. An example matrix of images used to systematically investigate the effect of drift in microscope imaging conditions with automated microstructure measurements - in this case to investigate the effect of working distance; (a) the acquired images, (b) edge differentiated images, used for ILS measurements, and (c) Otsu threshold images, all from the same field of view. The circled examples are referred to in the text. 


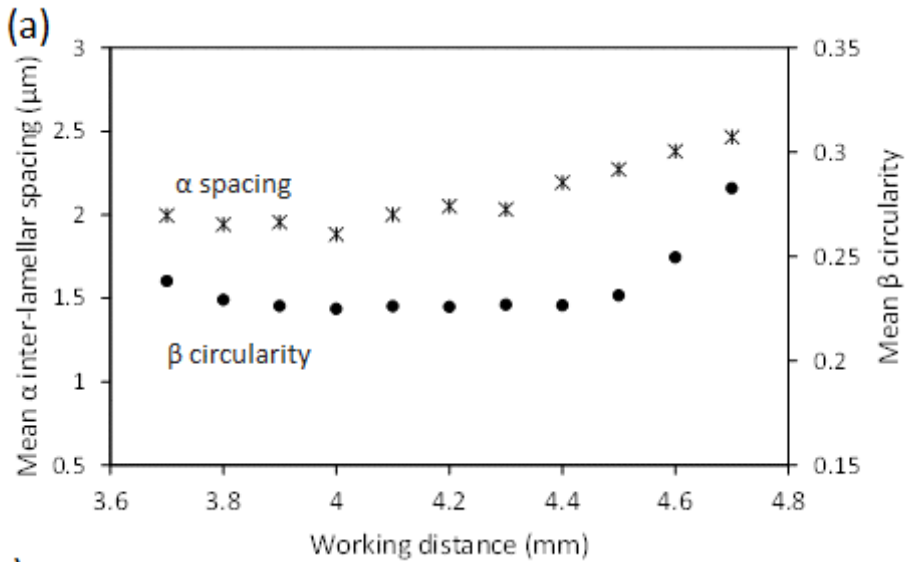

(b)

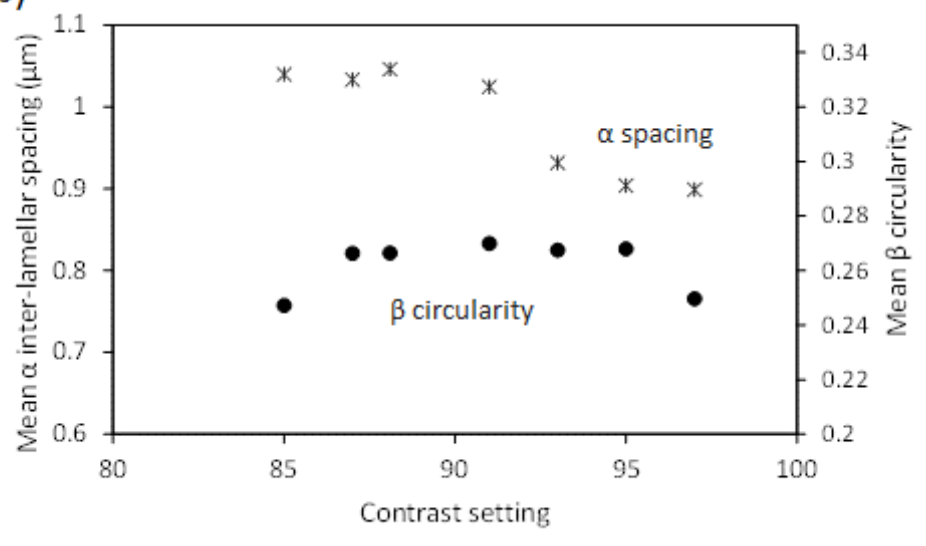

Figure 7. Effect of drift in key microscope variables on automated measurements of the interlamellar spacing (ILS) and $\beta$ phase circularity, showing the effect of; (a) the working distance and (b) contrast with brightness compensation.

Overall, this study has thus shown that with the current microscope and optimised settings the automated image processing method developed is sufficiently tolerant to drift in the key variables to allow accurate measurements over large stage translations and image mapping durations. The errors due to instrument drift within the ranges achievable in normal operation are low $(<3 \%)$ and only need to be considered when attempting to measure very small microstructural changes.

\subsection{Validation and effect of microstructure}

To check the accuracy of the measurements across different characteristic types of AM transformation microstructures, automated measurements were made on selected images representing the range of microstructures of interest and compared to manual measurements on the same images. Typical microstructure morphologies used are shown in Figure 8, which include (a) fine and (b) coarse basket weave, and (c) fine 'colony' $\alpha$, from the WAAM sample, as well as lightly spheroidized coarse, (d), fine (e), and severely spheroidized EBM microstructures (f). A direct comparison of the manually measured average $\alpha$ plate lamellar spacings with the results obtained from the automatic ILS analysis from the same images is provided in Figure 9. 
It should be noted that the range of average inter lamellar lath spacing's from image tiles in all the samples was limited to $0.5-2 \mu \mathrm{m}$, which demonstrates why an accurate user-independent measurement procedure is required to detect small changes in samples. Nevertheless, it can be seen that there is very good agreement between the manual and automated measurements for the data sets, comprising the WAAM and the less spheroidized EBM images. The results are also in close agreement with manual measurements available in the literature from a range of authors (e.g. [12$14,20,28,41])$.
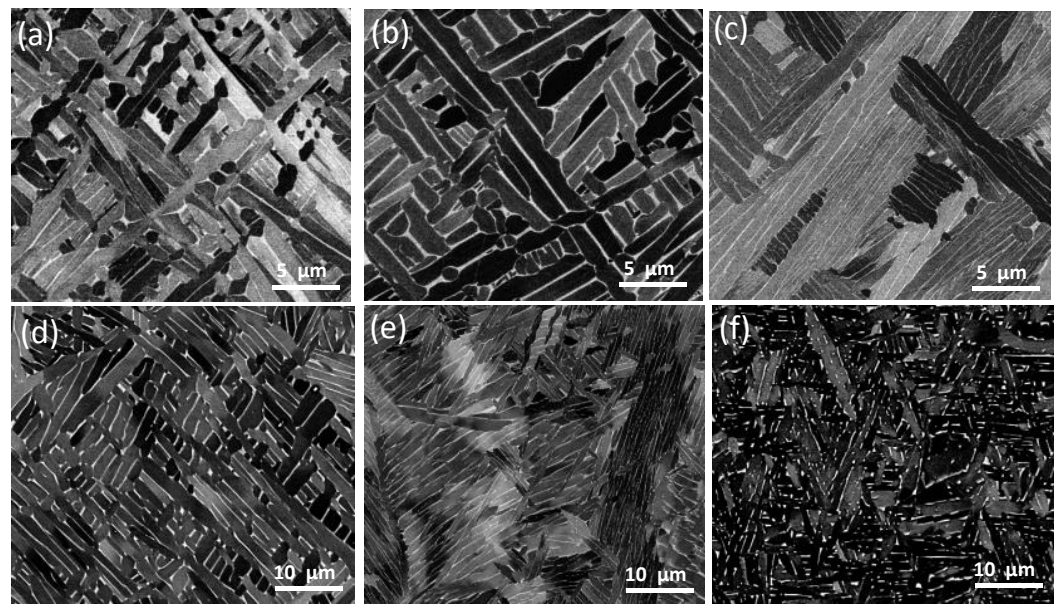

Figure 8. Example micrographs used to validate the automatic ILS measurements against a range of AM microstructures, through comparison with results obtained manually: (a) -(c); WAAM microstructures; fine basket weave, coarse basket weave and fine colony $\alpha$; (d)- (f) EBM samples, coarse and fine $\alpha$ and heavily spheroidized $\beta$, respectively.

However, a systematic error can be seen where the WAAM data falls slightly above the line of equivalence and the EBM below. In addition, the EBM samples with heavily spheroidized microstructures fall more significantly below the manually measured values. The small positive deviation of the WAAM data, which always contained continuous high contrast vanadium-rich $\beta$ layers between the $\alpha$ laths, could be caused by a slight oversensitivity of the edge function. In contrast, with the EBM results, it is apparent that spheroidization of the interlamellar $\beta$ phase effects the ILS measurements when the inter-lamellar $\beta$ becomes discontinuous. This is because the contrast defining the $\alpha$ lath boundaries in the BSE images is generated primarily by the composition difference between the $\alpha$ and $\beta$ phases; i.e. a continuous $\beta$ layer is required to reliably detect the $\alpha$ lath edges in BSE images. Thus, in microstructures such as in Figure 8(f) a significant error arises, that depends on the degree of spheriodization, where the linear intercept grid intercepts $\alpha-\alpha$ lath boundaries between discontinuous $\beta$ and without human intervention there is insufficient contrast to register the presence of an interface. From Figure 9 it can be seen that this error can be as great as $25 \%$ in heavily spheroidized microstructures. This problem does not arise in the WAAM samples where a $\beta$ layer was always seen between the $\alpha-\alpha$ laths. For this reason, when analysing the EBM samples 
automated ILS measurements were always performed in conjunction with $\beta$ circularity obtained made from the binarized images.

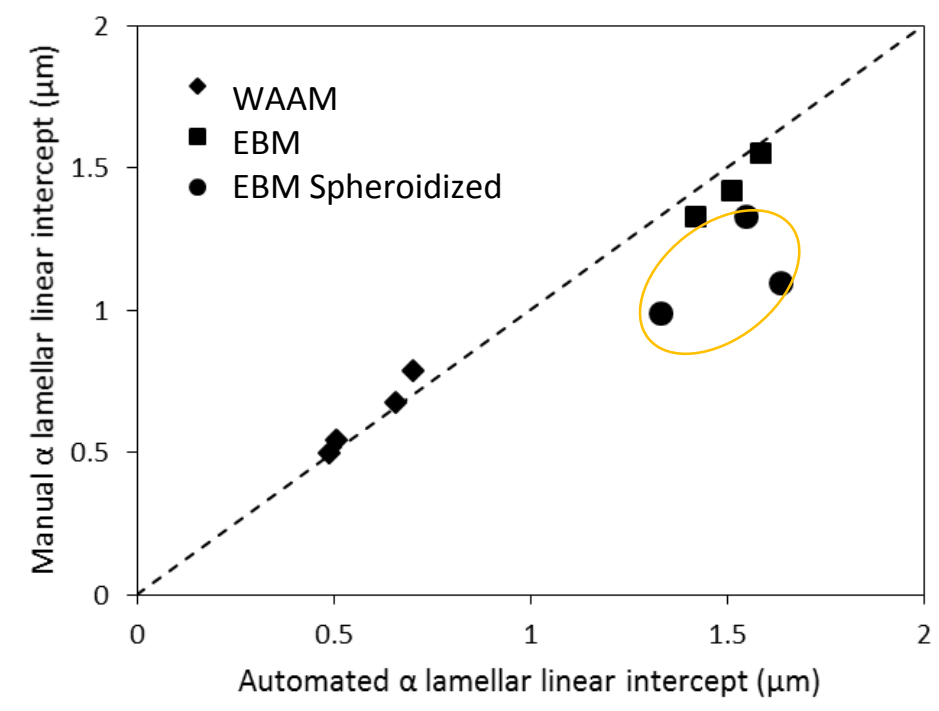

Figure 9. Comparisons between automatic quantification of the average $\alpha$ plate spacing and manual validation data for sets of different $\alpha$ morphologies from the EBM and WAAM samples. The data circled is from spheroidized EBM microstructures.

\subsection{Application}

The methodology described above was first applied to the EBM sample sets (Figure 1), using either automatic image acquisition with a regular spaced grid, to determine component scale variation at the microstructural scale, or a continuous set of image tiles to measure shorter-range banding effects. To further test the versatility of the automated mapping technique, results from samples produced by WAAM are subsequently presented, as this process operates at the other end of the build rate and component-scale spectrum. In this paper analysis of the WAAM samples has been confined to quantifying the microstructure gradients seen within the HAZ produced by individual beam passes. A more complete analysis of longer range effects will be presented elsewhere.

\subsection{Component Scale Microstructure Heterogeneity in EBM}

\section{Data mapped from the Chamber Height Rectangular Bar}

The $\sim 275 \mathrm{~mm}$ high, square section, sample built to the height of the chamber (Figure 1 a) was sectioned at $50 \mathrm{~mm}$ intervals, starting at a height $25 \mathrm{~mm}$ above the base plate, to ensure stable conditions had been reached. An array of 6 x 60 image tiles was mapped across the centre line of each section with an incremental spacing of $0.25 \mathrm{~mm}$. The data averaged across each section from 360 image tiles at each section height is shown in Figure 10 (a), with example images taken from the centre of each section at build heights of $25 \mathrm{~mm}, 175$ and $275 \mathrm{~mm}$ shown in Figure 10 (c). It should 
be noted that in Figure 10 (a) the first $25 \mathrm{~mm}$ of the bar, which is close to the build plate, has been ignored so that the sample would normally be considered to be in the 'steady state' height range of the chamber. The effect of closer proximity to the build plate will be discussed further below with the second sample set. The automated measurements in Figure 10 (a) suggest that in the 'steady-state' regime the $\alpha$ plate spacing's are very consistent with the build height, staying within a range of 1.5 $1.6 \mu \mathrm{m}$ except for the last data point near the top of the test piece, where an abrupt drop is seen. These average ILS measurements compare favourably to manual measurements performed by Hrabe and Quinn [14], although the results are slightly larger than the average lamellar spacing's they report of $\sim 1.2 \mu \mathrm{m}$ when using the same Arcam Process theme (i.e. 19). However, it should be noted a strong influence of the chamber working temperature has been reported [12], which was greater in the samples produced for our investigation $\left(740{ }^{\circ} \mathrm{C}\right.$ as opposed to $700{ }^{\circ} \mathrm{C}$ [14]). The last lower data point was obtained from a build height of $275 \mathrm{~mm}$ which was in close proximity to the top surface of the sample, as the test sample had in fact stopped being built near this position when the machine ran out of powder. The origin of this effect will be discussed further below.
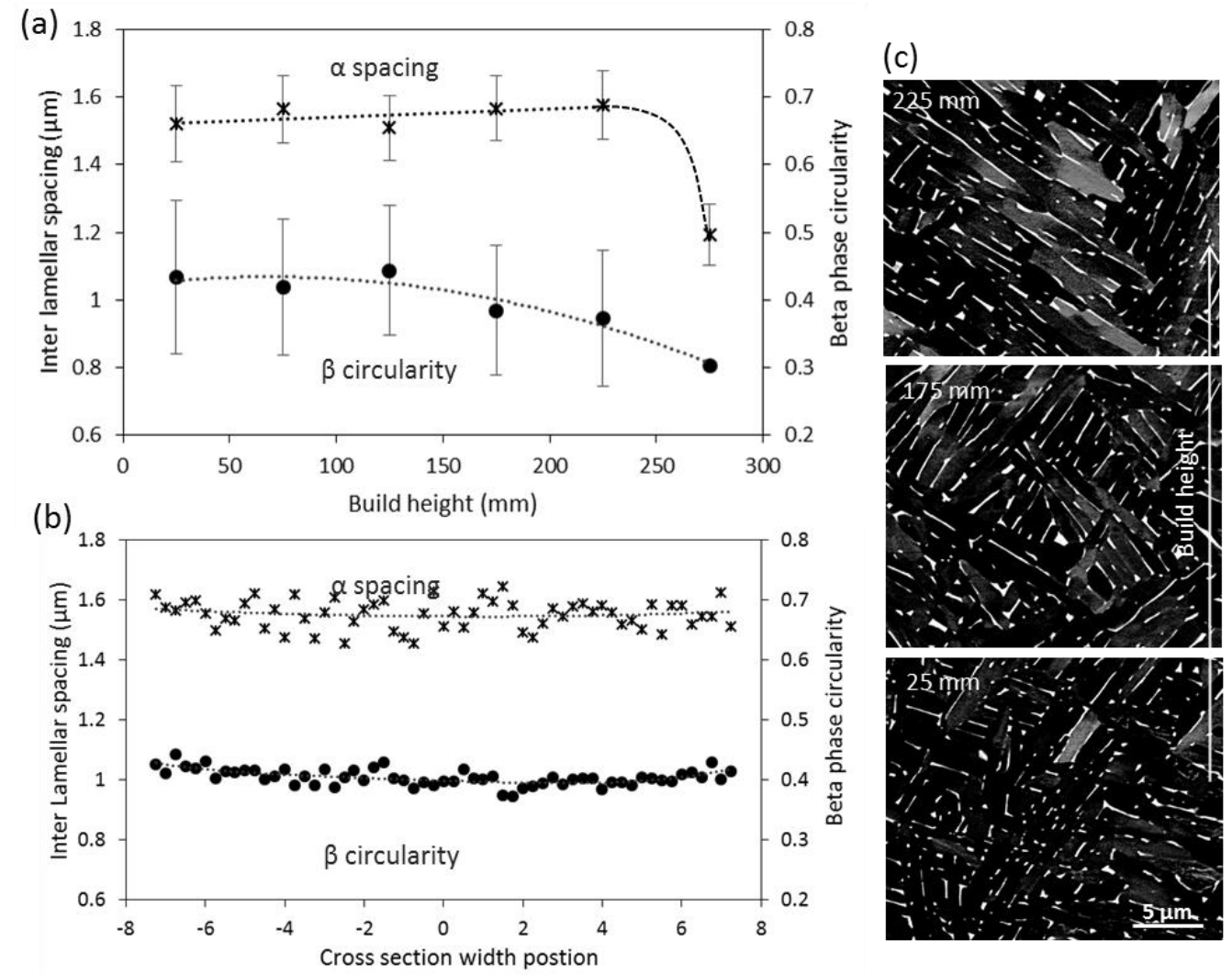

Figure 10. Measurements on the $\sim 280 \mathrm{~mm}$ high square section EBM sample built to the full height of the chamber; (a) average data from 360 measurements across sections at $50 \mathrm{~mm}$ intervals, starting at a height of $25 \mathrm{~mm}$ above the base plate, (b) measurements averaged from a 6 tile wide strip taken across each cross section apart from the last (which had a distinctly different ILS) and (c) example SEM image tiles taken from the canter of sections at build heights of $25 \mathrm{~mm}, 175$ and $275 \mathrm{~mm}$, showing different levels of $\beta$ spheroidization. 
If the final ILS point is ignored in Figure 10 (a), a linear regression fit to the data reveals a drift showing a progressive increase in the $\alpha$ lath spacing towards the top of the build of less than $5 \%$. While less reported in comparison to the $\alpha$ interlamellar spacings, Figure 10(a) further reveals a more significant variation in the degree of $\beta$ spheriodization. The extent of $\beta$ spheriodization can also be seen to decrease qualitatively in the example images in Figure 10 (c) towards the top of the built part and in Figure 10 (a) the $\beta$ phase average aspect ratio was measured to decrease by $~ 30 \%$ with build height. A similar effect can also be seen qualitatively in the results of Tan et al. (Figure 10 in ref. [13]) across a smaller build height [24]. In comparison the data plotted in Figure 10 (b) across the sample width, which was averaged across all the sections, apart for that at the highest position of $275 \mathrm{~mm}$ where the inter lamellar spacing deviation was noted, shows that both the inter-lamellar spacing and $\beta$ circularity were very uniform across the sample section.

The greater change measured in the $\beta$ phase circularity in comparison to the $\alpha$ lath spacing with build height can be readily attributed to the time at temperature, which is inversely dependent on the build height. For example, the sample studied here took 37 hours to produce, while a working temperature of $740{ }^{\circ} \mathrm{C}$ was maintained in the build chamber. This means the material near the bottom of the part was effectively heat-treated for a far longer period, leading to greater spheriodization of the $\beta$ phase, whereas material near the top would have been exposed to the build temperature for a shorter time (e.g. 7 hrs at a height $225 \mathrm{~mm}$ high). Over this build time range, the extent of breakup of the inter- $\alpha \beta$ phase into a non-continuous layer increased with greater thermal exposure and therefore reduced with build height (Figure 10 (a) and (c)).

As discussed above ( $\$ 3.4)$, the automated image processing of BSE images can lead to a positive error in the ILS data owing to spheroidization of the $\beta$ phase, which helps define the $\alpha$ plate boundaries from its higher $\mathrm{Z}$ contrast. This would lead to a systematic error in the $\alpha$ lamellar spacing measurements with increasing $\beta$ spheroidicity, so that the actual increase in ILS with build height in the 'steady state' range where the plot is relatively flat (from $25 \mathrm{~mm}$ to $225 \mathrm{~mm}$ in height) is greater than that automatically measured. By using the degree of spheoridization at each position to estimate the error (e.g. from the manual measurements in Fig. 9) it can be determined that the ILS measurements at the bottom of the build would be subject to a $\sim 15 \%$ greater absolute error than at the highest position in the 'steady state' range in Figure 10 (a). If this effect is taken into account, then the drift in $\alpha$ ILS with build height would be greater than that measured using the automated mapping technique and the real increase would have been of the order of $20 \%$, rather than $5 \%$.

Other authors have noted an increase in lath spacing with build height, based on image analysis with binarized images, but their data tends to focus on samples with a small build height and at positions starting closer to the build platform, where the higher rate of heat extraction would be expected to have a more significant effect on the cooling rate [13, 14, 32]. Galarraga et al. [32] have also reported a small change in manual measurements of the ILS and the tensile properties of EBM Ti64 samples with build height. In comparison to the rate of $\beta$ phase spheriodization, the $\alpha$ laths are not 
expected to coarsen as significantly from long exposures to the build temperature $\left(740{ }^{\circ} \mathrm{C}\right)[32]$ and their spacing is therefore more closely related to the extent of coarsening that arises from short duration thermal cycling to high temperatures below the $\beta$ transus, within the HAZs from thermal field overlap in subsequent repeated beam passes (see below) [26]. For the EBM process, with a simple part geometry, overall these results thus suggest the transient thermal conditions that generated the transformation microstructure were relatively constant with build height and this resulted in only a small variation in the inter-lamellar plate spacing.

\section{Data Mapped from the Prism-Shaped Samples}

To investigate the effect of changing the sample orientation and cross sectional area in a component, discrete image tile maps with a $200 \mu \mathrm{m}$ spacing were acquired across the mid triangular section of the prism-shaped test sample shown in Figure 1. The BSE images were again processed using the automated microstructure analysis software described in \$3.0. The $\alpha$ inter-lamellar spacing and $\beta$ phase circularity results from these samples are shown as $2 \mathrm{D}$ 'heat' maps in Figure 11. Data from the image maps has also been averaged in the width direction across a strip 9 tiles wide and plotted from base to apex for samples P2, V1 and H1 in Figure 12, to compare the behaviour of a prism sample with the same shape when built in different orientations. It should be noted that the number of tiles averaged from the $2 \mathrm{D}$ grid for each point diminished by necessity with sample section width from $2 \mathrm{~mm}$ below each tip as the sample became narrower, which increased the scatter. In the 2D maps some pixels have also been omitted at edges and corners where the data has been discounted due to metallographic polishing issues affecting the results.

When comparing the inverted prism samples P1-P3 in Figure 11, it can be seen that all the maps show a broadly similar behaviour despite having different rates of cross sectional area change with build height. The samples P1-P3 had a small point of contact with the base plate and were built outwards at different rates using support structures where required (see Figure 1 (b)), so that, heat transfer into the base plate was poor. This lead to a significantly larger average lamellar spacing of $1.4 \mu \mathrm{m}$ and a higher level of $\beta$ circulatory than for the other sample orientations. In contrast, when the same geometry of prism P2 was built either vertically (sample V1) with its square base or horizontally with a triangular side (sample H1) in contact with the base plate it had much better thermal contact to the build plate, which led to a marked overall reduction in the average interlamellar spacing to $1.0-1.2 \mu \mathrm{m}$, owing to a higher heat flux into the substrate. The data in Figures 11 and 12 also reveals more subtle effects similar to those discussed in relation to the $300 \mathrm{~mm}$ tall square section sample. 
(a)

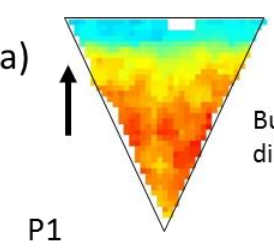

P1
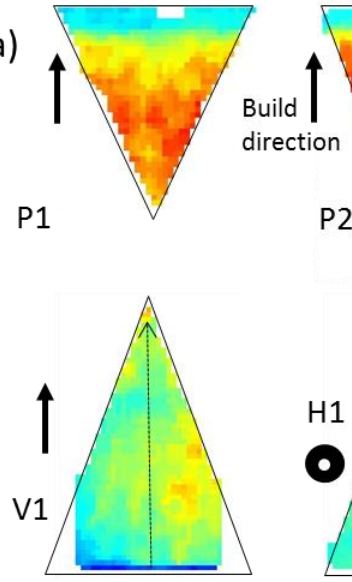

P2

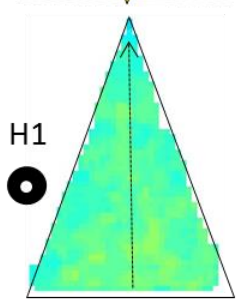

(b)
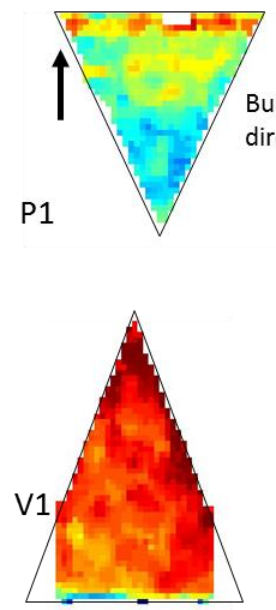
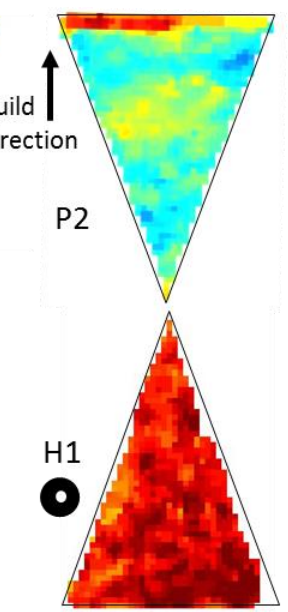
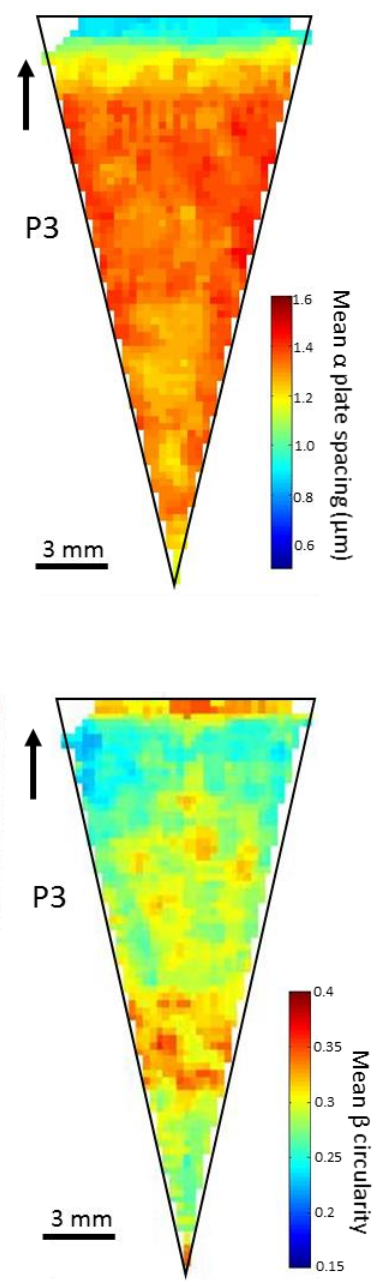

Figure 11. 2D plots showing the (a) Inter-lamellar spacing and (b) $\beta$ phase circularity results obtained from discrete image tile maps across the mid triangular section of the prism-shaped test samples, with different heights and build orientations. Note; the arrow across samples P1, V1, and H1 indicate the direction of plotting in Fig. 12. (Figure 1).

Of the sectioned views analysed, sample $\mathrm{H} 1$ had the most uniform microstructure, because it was orientated in the plane of the powder bed. However, the few data points near the apex of its section showed an unusually large lamellar spacing (Figure 12). The samples built apex down (P1-3) all showed a gradual increase in inter-lamellar spacing with height, which then became uniform before reducing more suddenly near the top surface, with a similar transient behaviour to that noted above for the tall rectangular test piece in Figure 10(a). Samples P1-P3 also tended to initially show an increase in $\beta$ phase circularity with height above the base plate, but in the tallest sample (P3) from above a build height of $\sim 12 \mathrm{~mm}$ the $\beta$ phase circularity then started to noticeably decrease. This indicates that at the bottom of each test piece the closer proximity to the base plate and the thinner section is reducing the effective background temperature by heat loss through the powder bed to the base plate. This reduced the $\alpha$ plate spacing and degree of spheroidization, despite the greater build time (i.e. the time in chamber while processing), up to a certain height of $\sim 12 \mathrm{~mm}$ 's. For greater build heights the degree of spheroidization then reduced again owing to the more dominant effect of 
the reducing build time on $\beta$ spheroidization, but the $\alpha$ spacing was relatively stable similar to in the tall rectangular build in Figure 10, until very close to the build top surface where there was again a sudden drop in $\alpha$ lamellar spacing.

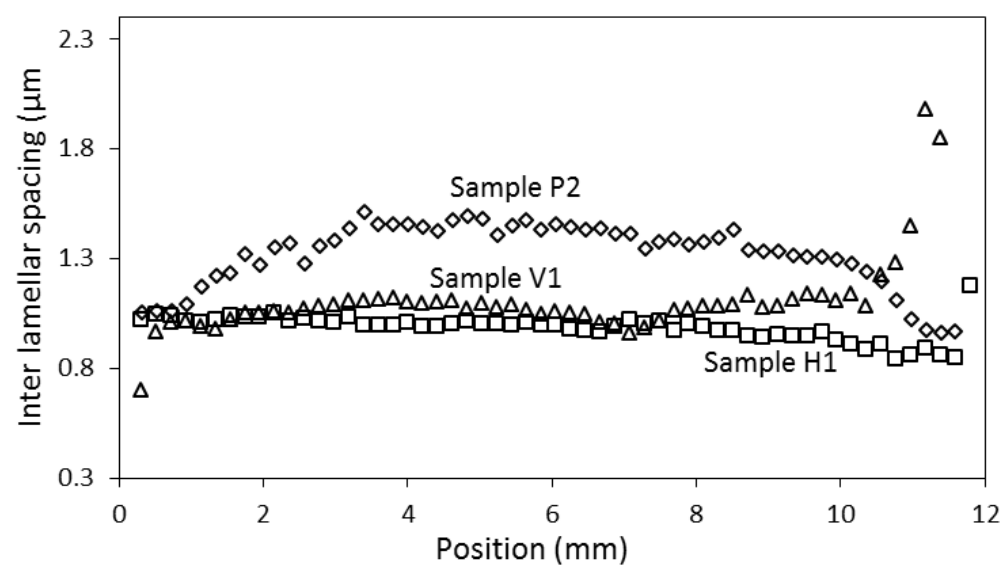

Figure 12. Average inter-lamella spacing data taken from the image maps (averaged in the width direction) plotted from base to apex for the same shaped prism samples, when built either base up (P2) base down (V1) or horizontally (H1), from a 9 tile wide strip (positions shown by the dashed arrows in Figure 11a).

As well as having an overall smaller average $\alpha$ lamellar spacing, sample V1 exhibited a more dramatic decrease in the lamellar spacing close the build plate, which was more sudden than for the inverted samples (P2). This again reflects the higher chill effect of the substrate with which it had a better thermal contact owing its square base. This sample also showed anomalously large interlamellar spacings close to its apex (Figure 12), similar to that seen for the H1 sample. It should be noted we have less confidence in the data for the apex position owing to the low number of image tiles averaged from the grid and issues with sample preparation (e.g. flatness) but the results suggest there may be an excess of energy applied by the EBM machine in small section locations at the apex of vertical or horizontal triangular sections.

The microstructure seen in the transient region noted at the top of all the builds that finished with a flat surface is highlighted in Figure 13, taken from sample P1. This layer of much finer inter-lamellar spacing was found at the top of all the inverted prism samples. In Figure 13 (a) it can be seen that there is a greatly diminished $\alpha$ lamellar spacing of less than $1 \mu \mathrm{m}$ within the $100 \mu \mathrm{m}$ of the build surface, or in the last two layers. However, martensite was not observed as vanadium enriched $\beta$ can clearly be in the image closest to the top surface. The very fine $\alpha$ spacing in this thin top build surface layer then increased back to a 'steady state ILS of $\sim 1.5 \mu \mathrm{m}$ at a depth of $\sim 1 \mathrm{~mm}$, as shown in Figures 11(a) and 12. This layer also gave anomalously high $\beta$ circularity values (Figure 11 (b) P1 $-\mathrm{P} 3)$ because with the very fine $\alpha$ spacing at the standard mapping image resolution the interlamellar $\beta$ appeared less continuous (Figure 13 (a)). 
Previously, other authors have reported the appearance of martensite, or partially martensitic microstructures in the top layer of EBM samples [12,18]. When the P1 prism sample investigated was completed, building continued to produce taller test pieces present in the chamber and the build cycle was run for several hours longer than that required to produce all the prism samples. This would have resulted in continued time at temperature tempering of any $\alpha$ ' to form $\alpha$ and $\beta$. For example, studies of tempering kinetics for Ti64 suggest $\alpha^{\prime}$ decomposition is complete in less than 30 minutes at temperatures above $700{ }^{\circ} \mathrm{C}$ [27]. At face value it is thus not surprising that the $2 \mathrm{D}$ mapping results reveal a similar fine 'tempered martensitic' transition region at the top of all the inverted prism samples, but it is not so obvious why this region does not then coarsen sufficiently to 'catch up' with the material deeper in the build if the machine continued in operation for several hours after the prism samples were built. Our measurements below, however, show that the average ILS in the bulk of the EBM samples was greater than in the WAAM materials (Figure 8) despite the much higher cooling rate [7], which suggests that the very fine $\alpha$ initially formed in the top layer rapidly coarsens with depth after transformation and this coarsening response largely controls the inter-lammelar spacing seen in the majority of a build.

The explanation for this behaviour is that the $\alpha$ lamellar spacing coarsens rapidly when it is exposed to temperatures within the range of $\alpha-\beta$ transus approach curve (i.e. when the $\alpha$ volume fraction starts to reduce, as $\beta$ re-grows) and this typically requires temperatures to be reached higher than that of the background build temperature. For example, in work on directed energy processes it is noted that $\alpha$ starts to coarsen once its 'dissolution' temperature has been exceeded (e.g. from $700-990{ }^{\circ} \mathrm{C}$ ) [26,42] (see $\$ 4.3$ below). The fineer $\alpha$ transition layer seen in Figure 13, and recorded in the statistical data in Figure 12, is thus retained because in the EBM process there is a very high cooling rate when $\alpha$ first transforms and this microstructure does not coarsen readily below $750{ }^{\circ} \mathrm{C}$. With repeated beam passes from additional added layers, the $\alpha$ phase will transform back fully to $\beta$ several times to a depth equivalent to several added layers [7,9]. Although the reducing peak temperature experienced by a given volume of material in each subsequent heating cycle with each successively added layer will tend to lower the cooling rate through the $\beta$ transus, which would increase the $\alpha$ inter-lamellar spacing, the cooling rate is still much higher in EBM compared to the WAAM process, and this produces a finer final $\alpha$ plate spacing near the top of the part (see Figure 9). However, in EBM the background temperature is already at the bottom end of the temperature range where rapid $\alpha$ coarsening is expected (e.g. $>740{ }^{\circ} \mathrm{C}$ ). There will, therefore, be a high level of thermal field overlap in the critical range for $\alpha$ phase coarsening from repeated beam passes as scanning continues and further layers are added [7,43]. Overall, this suggests the top surface transition region from fine $\alpha$ to the coarser $\alpha$ microstructure seen in the EBM samples, is predominantly caused by re-heating in the heat affected zone from overlapping beam tracks to temperatures above the build temperature, but below the $\beta$ transus, rather than from the long term exposure experienced at build chamber 
background temperatures of $700-740^{\circ} \mathrm{C}$. As clearly shown above (e.g. Figure 10) long term exposure of parts at build chamber temperatures does however cause spheriodization of the interlammelar $\beta$ phase within the transformation microstructure and these two effects are therefore to some extent de-coupled.

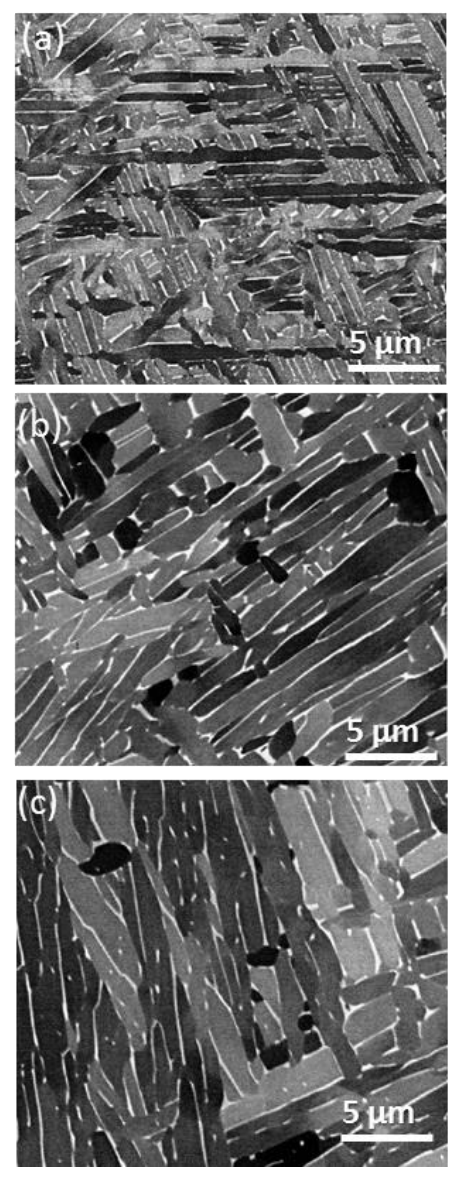

Figure 13 Example BSE images taken from the top layer of prism sample P1, at depths of (a) 0.1 $\mathrm{mm}$, (b) 1.3 and (c) $2.5 \mathrm{~mm}$.

\subsection{Layer Scale Microstructure Heterogeneity in the EBM process}

With the EBM powder bed process, due to the small layer height (typically $~ 50 \mu \mathrm{m}$ ), high level of thermal field overlap from neighbouring beam passes, orthogonal rotation of the raster pattern between layers, and long exposure times at high build temperatures, layer wise microstructural banding in Ti64 parts is a relatively weak effect, difficult to detect, that has not been widely commented on in the literature. Nevertheless, weak differential etching between layers can be apparent in optical images under certain conditions (Figure 14). As this contrast tends to be stronger with parts built with older generation machines, the sample used for study here was produced with an Arcam S12 machine using standard titanium build parameters [11]. Automated image acquisition and quantification was applied to the centre hatch region of the sample in the vertical plane. This 
involved acquiring a 12 tile wide set of stitched SEM images over a build height of $1.5 \mathrm{~mm}$, which would be expected to include $~ 25$ layers. In Figure 14 (a) contrast from differential etching can be seen from an enlarged macro lens image, which was hard to distinguish with higher magnification optical microscopy. A corresponding SEM image tile subset is also provided in Figure 14 (b) that extends $100 \mu \mathrm{m}$ in the build direction and is thus over one layer high. The ILS and $\beta_{\text {Circ. data }}$ averaged in the plane of the build obtained from the full image map is shown in Figure 9 (c).

The higher magnification SEM images extracted from the map area (Figure 14b) show that the microstructure is typical for the EBM process [7-14,19], being predominantly basket weave lamellar $\alpha$, with partially spheroidized $\beta$, and is very homogeneous; to the extent that it is difficult to detect any systematic microstructure variation across an added layer by eye. The microstructure quantification results presented in Figure 14 (b) also show insignificant long range variation in both the $\alpha$ inter-lamellar spacing and the $\beta$ phase circularity across the $1.5 \mathrm{~mm}$ build height analysed. However, it can be seen that there is considerable local scatter in the data which may obscure weak periodicity in the ILS measurements. The ILS data was therefore processed further using a Fourier transform in an attempt to reveal a characteristic frequency. It can be seen from Figure 14 (d) that discrete Fourier transformation successfully extracted a dominant periodicity from the original data with a wavelength of about $60 \mu \mathrm{m}$, which is consistent with the expected layer height in this sample. Therefore, despite the long term exposure at the build temperature the sample still had a weak memory of the original HAZ layering. In comparison, no periodicity was detected in the $\beta$ circularity data, which is not that surprising as the extent of spheroidization mainly depends on the exposure time and background build temperature and this would not be expected to vary greatly in a small area of material. Although detectable, the local difference in $\alpha$ lamellae spacing was very small with the max amplitude in ILS being only $\sim 0.15 \mu \mathrm{m}$, or $7 \%$ of the average, which is probably negligible in terms of its effect on mechanical behaviour, and reflects the high level of homogeneity of the transformation microstructure produced by the EBM process. 


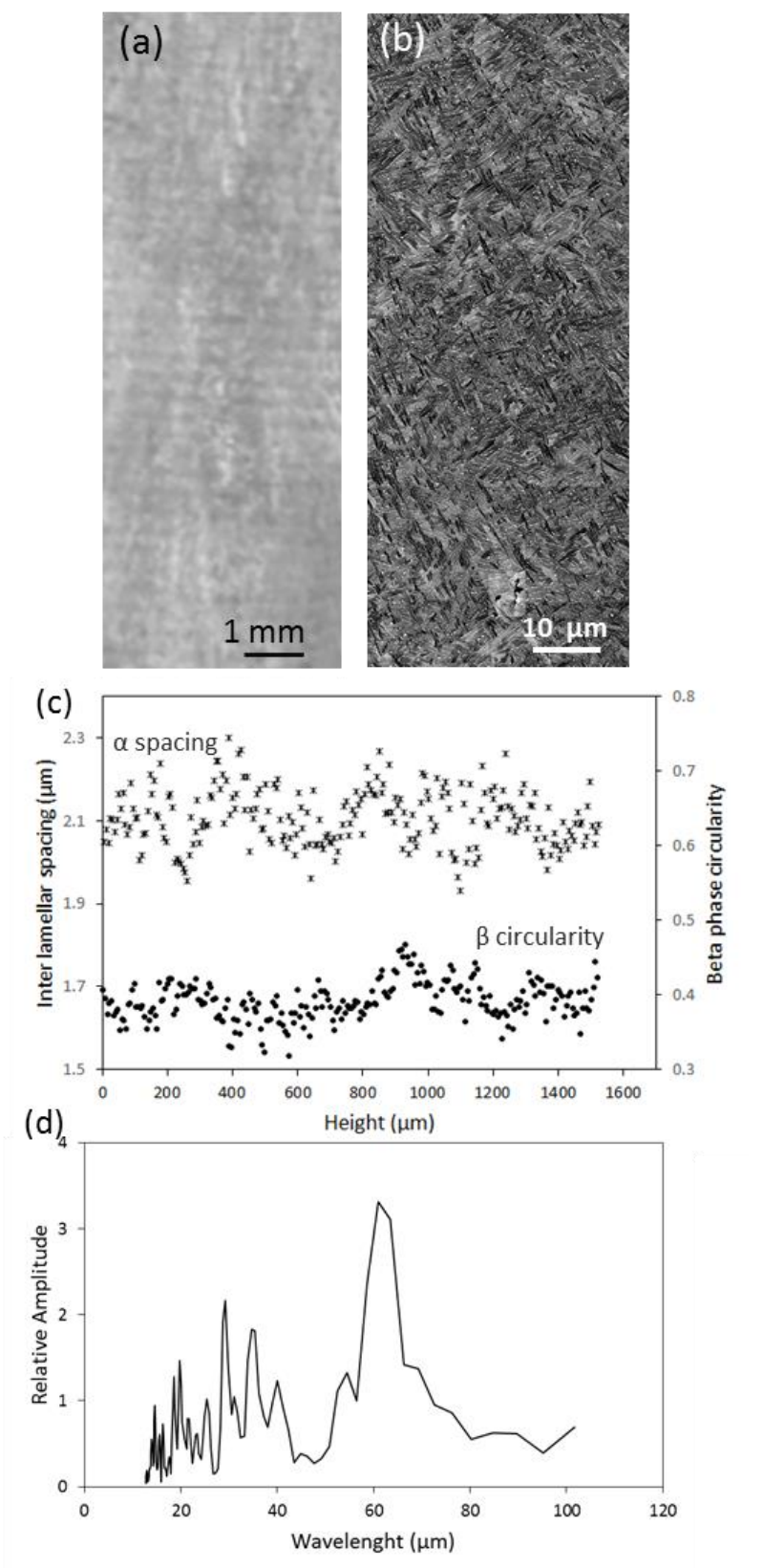

Figure 14. The automated image acquisition and quantification method applied to detecting short range microstructure layer banding in the EBM process; (a) optical macro image showing faint etched layer bands, (b) a SEM high resolution tile map over $\sim 3$ layers, (c) the mean $\alpha$ inter lath spacing (ILS) and $\beta$ circularity as a function of height, (d) a Fourier transform of the ILS data plotted as a function of wavelength.

\subsection{Application to WAAM HAZ Banding}

High deposition rate directed energy AM processes like WAAM operate with a much more diffuse heat source and larger layer height $(1-2 \mathrm{~mm})$ which results in lower cooling rates $\left(\dot{T}=10^{1}-10^{2} \mathrm{~K}\right.$ $\left.\mathrm{s}^{-1}\right)[10,17]$ such that a Widmanstätten $\alpha$ microstructure normally forms directly from the $\beta$ phase on cooling below the $\beta$ transus [20,21]. The thermal field from each deposition pass is also larger and 
overlaps less strongly than in the EBM powder bed process, resulting in more obvious heat affected zones in deposits (Figure 15 (a)) [20,21,24,26]. In a typical WAAM deposit it has been noted that the first HAZ band appears at a position below the top surface where the peak temperature rise is equivalent to the $\beta$ transus temperature (Figure 15 ) $[20,21,24,26]$. In a single pass wide wall at this depth modelling has shown that the isotherms generated by the heat source are approximately parallel, due to the combination of the high part aspect ratio geometry and large temperature interval between the melting point and $\beta$ transus $\left(\sim 700{ }^{\circ} \mathrm{C}\right)[10,19]$. As more layers are deposited, the heat source increments upwards and this leads to further regular, linear, HAZ bands being generated within the steady state portion of the build with a spacing equivalent to the layer height. The region above the first HAZ band is typically equivalent to 4- 5 added layers high and is fully $\beta$ heat-treated in the final pass so that it transforms fairly uniformly on cooling to produce a Widmanstätten basket weave microstructure [20,21]. The HAZ bands are thought to be associated with the temperature interval above which the $\beta$ phase volume fraction starts to increase significantly $\left(\sim 750{ }^{\circ} \mathrm{C}\right.$ [42]), which leads to rapid $\alpha$ phase coarsening and a gradient in the inter-lamellar spacing with build height $[19,20,26,29]$. Morphological and local texture changes have also been noted across the HAZ bands and attributed to specific combinations of peak temperature, time at temperature, and cooling rate although the microstructure gradient produced has not yet been quantified [19,20,24,26].

To characterise the behaviour of the transformation microstructure seen in the WAAM process [20,21] over the distance required to quantify the repeat pattern found across a typical HAZ band, a BSE image tile map was produced as a continuous strip 200 tiles long by 5 tiles wide. This was sufficient to fully traverse the first HAZ band into the fully $\beta$ treated region at the top of the deposit. Following batch image processing, the ILS values obtained were averaged in the width direction and are shown in Figure 15 as a function of build height. As the transformation microstructure in the WAAM samples had a continuous inter-lamellar layer of retained $\beta$, measurement of the $\beta_{\text {Circ }}$ is not relevant and was therefore not attempted.

By accurately quantifying the $\alpha$ phase lamellar spacing, automated mapping can be seen in Figure 15 to have been successful in clarifying the detailed behaviour of the transformation microstructure across a typical HAZ band. In Figure 15 the $\alpha$ ILS can be observed to increase across the HAZ band from a background level of $0.7-0.75 \mu \mathrm{m}$ to a peak of $0.9-1 \mu \mathrm{m}$ in a 'saw tooth' wave-like fashion. In comparison, the top part of the wall has a relatively uniform inter-lamellar spacing, as it all transformed from the $\beta$ phase in the last pass with a similar cooling rate [10] and has not been subsequently re-heated. The background level between the first two HAZ bands shown is also similar to that seen in the fully $\beta$-annealed top proportion of the wall. Taken together, this data suggests that there was not sufficient overlap of the thermal field in the critical temperature range from $750{ }^{\circ} \mathrm{C}$ to the $\beta$ transus temperature [24,26] to cause substantial coarsening of the $\alpha$ phase between the HAZ bands. However, the material where coarsening occurred still constituted a large proportion of each added layer (at least 70\%), and thus would be expected to alter the average mechanical properties of 
a bulk component. Further data is also needed to confirm if the same behaviour continues to repeat lower down in the sample below the first two HAZ layers; nevertheless, these preliminary results confirm that the average lamellar spacing for the WAAM process [20,41] is less than that for the EBM process [12-14], despite the lower cooling rate, but is more heterogeneous [7,10]. As discussed above, this divergence is caused by the more uniform and greater level of coarsening seen for the $\alpha$ phase in the powder bed process.

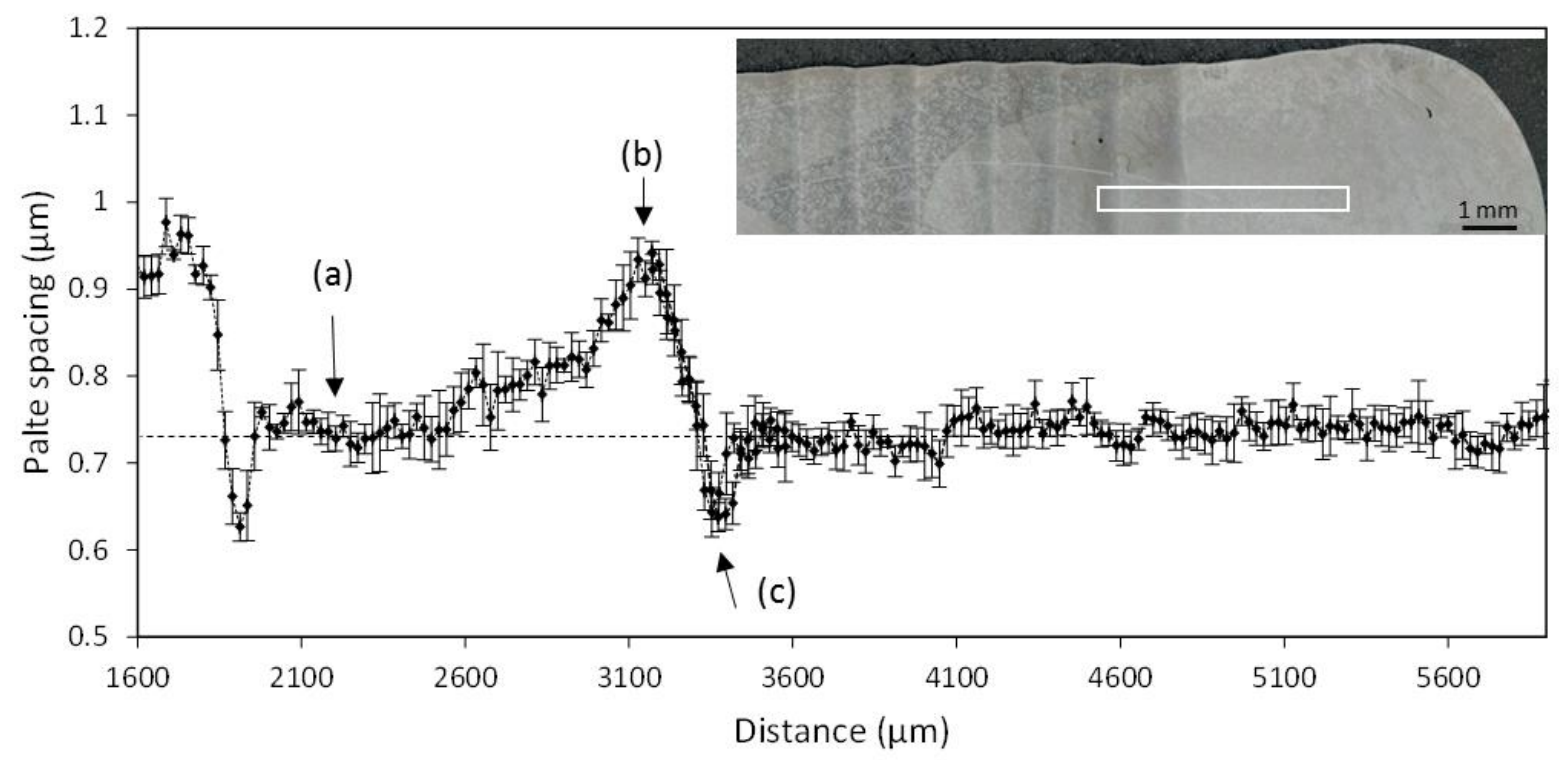

Figure 15 Characterisation of a single bead wide WAAM wall sample, showing quantification of the interlamellar spacing averaged from a 5 tile wide $(125 \mu \mathrm{m})$ stitched image strip, mapped across the last two HAZ layer bands into the fully $\beta$ heat treated region at the top of the wall. The position of the image tile map is shown to scale in the insert optical image. The arrows indicate the positions of the images shown in Figure 8 (a) - (c).

In addition, the ILS data in Figure 15 has revealed that the wave like behaviour across a HAZ band is more complex than just a microstructure coarsening response. It can be seen that the $\alpha$ lamellar spacing first rises at an increasing rate, as might be expected, but then drops abruptly at the top of the HAZ band, to fall below the background level to a minimum spacing of $\sim 0.6 \mu \mathrm{m}$ for a short distance (marked by arrow (c) in Figure 15) before rising again back to the background value. The thin transition layer where the spacing falls abruptly can also be correlated to a white etch line at the top of each HAZ band in the image insert in Figure 15. The exponential rise in $\alpha$ lamellar spacing (marked arrow (b) in Figure 15) with temperature is typical of a thermally activated process and mirrors the increase in $\beta$ volume fraction expected in the $\beta$ transus approach curve [42]. However, the dip in ILS measurements near the top of each HAZ band is surprising and warrants further investigation. The images presented in Figure 8 (a) - (c) to test the automated image analysis against manual measurements from a range of microstructures were selected from the positions indicated by the arrows in Figure 15. From these images it can be seen that this interesting behaviour can be related to 
a change in morphology of the $\alpha$ phase, which varies across each HAZ band from the fine background conventional basket weave microstructure (Figure 8 (a)), to a coarsened basket weave morphology, which has the maximum ILS spacing (Figure 8 (b)), to - in the region where the ILS values dip momentarily - a more 'colony-like' morphology consisting of larger packets of very finally spaced single $\alpha$ variants (Figure 8 (c)). The origin of this complex behaviour, which is associated with a narrow temperature interval below the $\beta$ transus temperature, will be the subject of a future paper. However, the analysis above confirms the original qualitative assessment of HAZ banding by Kelly and Kampe [24,26] who similarly reported a steep gradient in $\alpha$ lamellar spacing within each HAZ band and that the $\alpha$ lamellar spacing was smallest and had a colony-like morphology just above each layer band.

\subsection{Conclusions}

A microstructure quantification procedure has been successfully developed for analysing fine scale transformation microstructures in AM components over large length scales with good statistical accuracy. This was achieved by combining a mapping function with high resolution BSE imaging in an SEM with an in-house developed, automated, image analysis GUI implemented in Matlab. The image analysis tool was designed to batch process multiple image tiles using a pre-processing procedure to first dynamically correct background contrast variation and then quantify the $\alpha$ phase inter-lamellar lath spacing (ILS) through edge detection as well as the level of inter-lamellar $\beta$ spheroidization; both of which are important microstructural parameters that can be related to the local thermal history experienced in AM parts.

A series of tests were performed to validate the consistency of the quantification method with respect to experimental errors that could occur with large stage translations, such as changes in the SEM working distance and brightness and contrast levels. It was demonstrated that drift in such instrument parameters did not limit the quality of the data in a practical mapping application, with the maximum errors being of the order of $<3 \%$. It was also shown that when analysing BSE images spheroidization of the $\beta$ phase could create a positive systematic error in inter-lamellar spacing measurements.

The batch processing of image tile maps was used to explore both short and long range microstructure heterogeneity in two case studies. In powder bed electron beam melting (EBM) samples it was found that although the ILS was relatively homogeneous, a small increase was detected with build height $(<5 \%)$. In addition, more significant difference were observed in whole components as a function of changes to their thermal contact with the base plate and in the top $1 \mathrm{~mm}$ of a build a transition to a noticeably finer microstructure was also observed. In contrast, more marked changes were measured in the level of $\beta$ spheroidization $(\sim 30 \%)$. This could be attributed to the effect of prolonged thermal exposure at high working temperatures in the EBM chamber which varies inversely with build height. 
The automated microstructure mapping procedure has also been applied to characterize the microstructure gradients seen in HAZ banding in Wire Arc Additive Manufacturing (WAAM). This revealed a characteristic repeat behaviour in the ILS data across each added layer, where the $\alpha$ lamellar spacing rose at an increasing rate and then dropped abruptly, before falling sharply below the background level for a short distance at the top of each HAZ band. The expected exponential rise in $\alpha$ lamellar coarsening with temperature mirrored the increase in $\beta$ volume fraction on approaching the $\beta$ transus. However, the drop in the ILS measurement below the background level at the top of each HAZ band was found to be associated with a change in morphology of the $\alpha$ phase to a fine colony-like microstructure.

\section{Acknowledgements}

The authors would like to thank Dr F. Martina, Dr P.A. Colgrove, and Prof S.W. Williams from Cranfield University, UK, for the provision of the WAAM samples and GKN aerospace, UK for the EBM specimens. The authors are appreciative of the EPSRC's (grants LightForm - EP/R001715/1; NEWAM - EP/R027218/1), Innovate UK (Open Architecture Additive Manufacturing, OAAM) and the Metallic Systems CDT (EP/L016273/1) for supporting aspects of this research. Professor P.B. Prangnell is also grateful to the Royal Academy of Engineering, UK and Airbus Operations UK for financial support.

\section{Data availability}

The processed data required to reproduce these findings are available to download from doi:10.17632/jdvx4g2498.1

\section{References}

1. G. Lütjering, JC, Williams, Titanium, Springer, (2007). doi:10.1007/978-3-540-73036-1

2. B. Vayre, F. Vignat, F. Villeneuve, Mech. Ind. 13 (2012). 89-96

3. E. Atzeni, A Salmi, Int. J. Adv. Manuf. Technol. 62 (2012) 1147-1155.

4. Y. Zhai, D.A. Lados, J.L. LaGoy, JOM 66 (2014) 808-816.

5. T. DebRoy, H.L. Wei J.S. Zuback, T. Mukherjee, J.W. Elmer, J.O. Milewski, A.M. Beese, A. Wilson-Heid, A. De W. Zhang, Progress Mater. Sci. 92 (2018) 112-224

6. W. Xu, E.W. Lui, A. Pateras, M. Qian, M. Brandt, Acta Mater. 125 (2017) 390-400.

7. C. Körner, Int. Mater. Reviews, (2016) 61:5, 361-377

8. H. Galarragaa, D.A. Lados, R.R. Dehoff, M.M. Kirka, P. Nandwana, Additive Manu. 10 (2016) 47-57.

9. Shen, N. and K. Chou, (2012), ASME 2012 Int. Manuf. Sci and Engineering Conference, Notre Dame, Indiana, USA, June 4-8, 2012, MSEC2012-7253, pp. 287-295. 
10. V.D. Fachinotti, A. Cardona, B. Baufeld, O. Van der Biest, Acta Mater. 60 (2012) 6621-6630 11. A.A. Antonysamy, J. Meyer, P.B. Prangnell, Mater. Character. 84 (2013) 53-168.

12. S.S. Al-Bermani, M.L. Blackmore, W. Zhang, I. Todd, Metall. Mater. Trans. 41A (2010) 3422-34.

13. X. Tan, Y. Kok, Y.J. Tan, M. Descoin, D. Mangelinck, S.B. Tor, K.F. Leong, C.K. Chua, Acta Mater. 97 (2015) 1-16.

14. N. Hrabe, T. Quinn, Mater. Sci. Eng. 573A (2013) 264-270.

15. J. A. Koepf, M.R. Gotterbarm, M. Markl, C. Korner, Acta Materialia, 152 (2018) 119-126.

16. A. Lundbäck, L-E. Lindgren, Finite Elements in Analysis and Design 47 (2011) 1169-1177.

17. X. Baia, P.A. Colegrove, J. Ding, X. Zhou, C. Diao, P. Bridgeman, JR Hönnige, H. Zhang, S.W. Williams, Int. J. Heat Mass Transfer, 124 (2018) 504-516.

18. A. Safdara, L.-Y. Wei, A. Snis, Z. Lai, Mater Character. 65 (2012) 8 - 15.

19. P. Åkerfeldt, M-L. Antti, R. Pederson, Mater. Sci. Eng. 674A (2016) 428-437.

20. F. Martina, P.A. Colgrove, S.W. Williams, and J Meyer, Metall. Mater. Trans, 46A (2015) 6103-6118.

21. F. Martina, J. Mehnen, S.W. Williams, P. Colegrove, F. Wang, J. Mater. Proc. Technol. 212 (2012) 1377- 1386.

22. C. Guoa, W. Gea, F. Lina, J. Mater. Proc. Technol. 217 (2015) 148-157.

23. G. Lütjering, Mater. Sci Eng. 243A (1998) 32-45.

24. S.M. Kelly, S.L. Kampe. Metal Metall. Mater Trans. 35 (2004) 1861-1867.

25. B. Radhakrishnan, S. Gorti, S S Babu, Metall. Mater Trans. 47A (2016) 6577-6592.

26. S.M. Kelly, S.L. Kampe, Metall. Mater. Trans. 35A (2004) 1869-1879.

27. F.X. Gil Mur, D. Rodrguez, J.A. Planell, J Alloys Compounds, 234 (1996) 287-289

28. B. Baufeld, E. Brandl, O. van der Biest, J. Mater Proc. Technol. 211 (2011) 1146-1158.

29. E. Brandl, A. Schoberth, C. Leyens, Mater, Sci. Eng. 532A (2012) 295- 307

30. T. Scharowsky, V. Jüchter, R. F. Singer, C. Körner: Adv. Eng. Mater. 17 (2015) 1573-1578.

31. F.J. Gil , M.P. Ginebra, J.M. Manero, J.A. Planell, J. Alloys Compounds, 329 (2001) 142152.

32. H. Galarragaa, R.J. Warren, D.A. Lados, R.R. Dehoff, M.M. Kirka, P. Nandwana, Mater. Sci. Eng. 685A (2017) 417-428

33. J. Chrapoński, W. Szkliniarz, Mater. Character. 46 (2001) 149 - 154.

34. H. Li, Z. Ji, H. Yang, Mater Character. 76 (2013) 6 - 20.

35. N. Ostu, IEEE Trans Syst Man Cybern, 6(1) (1979) 62-66.

36. F. Wang, S. Williams, P. Colegrove, A.A. Antonysamy, Metall. Mater. Trans. 44A (2013) 968-977.

37. L. Reimer. (1985) Imaging with Secondary and Backscattered Electrons. In: Scanning Electron Microscopy. Springer Series in Optical Sciences, vol 45. Springer, Berlin, Heidelberg.

38. J. Canny, IEEE Trans Syst Man Cybern, 8(6) (1986) 679-698. 
39. L.S. Davis, Compu. Graphics Image Proc. 4(3) (1975) 248-270.

40. D. Marr, \& E. Hildreth. Proc. Royal Soc. B: Bio. Sci. 207 (1167) 187-217.

41. M. Neikter, P. Åkerfeldt, R. Pederson, M.-L. Antti, V. Sandell, Mater Chracter. (2018) in press.

42. I. Katzarov, S. Malinov, W. Sha: Metall. Mater. Trans. 33A (2002) 1027-40.

43. S. Tammas-Williams, H. Zhao, F. Leonard, F. Derguti, I. Todd, P. B. Prangnell, Mater Character. 102 (2015) 47-61. 


\section{Figure Captions}

Figure 1. The EBM sample sets studied by automated microstructure mapping; (a) a tall vertical square section sample and prismatic-shaped samples (b) P1-P3 built inverted with different wall angles and supports where required, and (c) the sample shape P2 built in different orientations; vertical V1, horizontal $\mathrm{H} 1$. The standard reference frame used $(\mathrm{x}, \mathrm{y}, \mathrm{z}$; where $\mathrm{x}-\mathrm{y}$ are the build plane and $\mathrm{z}$ is the build height direction) and planes the samples were sectioned in for analysis are indicated.

Figure 2 Examples of different automated image mapping strategies adopted for acquiring high resolution data over large areas, shown after mapping with carbon contamination indicating the image capture positions: (a) a large area 2D grid on an EBM prism sample, (b) a continuous 5 tile wide strip from a WAAM wall.

Figure 3. Examples of the effectiveness of image enhancement and post-processing showing: (a) a typical original SEM image of lamellar Ti-6Al-4V microstructure with strong background contrast variation, (b) an Otsu adaptive threshold image without pre-processing, (c) - (d) the pre-processing steps, (c) following application of a low-pass Gaussian filter and (d) after noise reduction and contrast enhancement. The final binarized Ostu [35] adaptive threshold image and edge detected images are shown in (e) and (f) respectively, for comparison with (a).

Figure 4. Comparison of the grayscale contrast seen in a typical image, which contains noise and background ( $\alpha$ phase) variation, with that after Gaussian low pass filter background subtraction and auto contrast enhancement.

Figure 5. Illustration of the Canny edge detection [38] method; (a) an enlarged differentiated image with (b) the final 'edged' image. By growing 'edges' from high to low contrast areas accurate results are obtained even in regions of low contrast (circled). Occasionally small faint isolated regions can still be missed by the procedure (arrows).

Figure 6. An example matrix of images used to systematically investigate the effect of drift in microscope imaging conditions with automated microstructure measurements - in this case to investigate the effect of working distance; (a) the acquired images, (b) edge differentiated images, used for ILS measurements, and (c) Otsu threshold images, all from the same field of view. The circled examples are referred to in the text.

Figure 7. Effect of drift in key microscope variables on automated measurements of the interlamellar spacing (ILS) and $\beta$ phase circularity, showing the effect of; (a) the working distance and (b) contrast with brightness compensation. 
Figure 8. Example micrographs used to validate the automatic ILS measurements against a full range of microstructures, through comparison with results obtained manually: (a) -(c); WAAM microstructures; fine basket weave, coarse basket weave and fine colony $\alpha$; (d)- (f) EBM samples, coarse and fine $\alpha$ and heavily spheroidized $\beta$, respectively.

Figure 9. Comparisons between automatic quantification of the average $\alpha$ plate spacing and manual validation data for sets of different $\alpha$ morphologies from the EBM and WAAM samples. The data circled is from spheroidized EBM microstructures.

Figure 10. Measurements on the $\sim 280 \mathrm{~mm}$ high square section EBM sample built to the full height of the chamber; (a) average data from 360 measurements across sections at $50 \mathrm{~mm}$ intervals, starting at a height of $25 \mathrm{~mm}$ above the base plate, (b) measurements averaged from a 6 tile wide strip taken across each cross section apart from the last (which had a distinctly different ILS) and (c) example SEM image tiles taken from the canter of sections at build heights of $25 \mathrm{~mm}, 175$ and $275 \mathrm{~mm}$, showing different levels of $\beta$ spheroidization.

Figure 11. 2D plots showing the (a) Inter-lamellar spacing and (b) $\beta$ phase circularity results obtained from discrete image tile maps across the mid triangular section of the prism-shaped test samples, with different heights and build orientations. Note; the arrow across samples P1, V1, and $\mathrm{H} 1$ indicate the direction of plotting in Fig. 12. (Figure 1).

Figure 12. Average inter-lamella spacing data taken from the image maps (averaged in the width direction) plotted from base to apex for the same shaped prism samples, when built either base up (P2) base down (V1) or horizontally (H1), from a 9 tile wide strip (positions shown by the dashed arrows in Figure 11a).

Figure 13 Example BSE images taken from the top layer of prism sample P1, at depths of (a) 0.1 $\mathrm{mm}$, (b) 1.3 and (c) $2.5 \mathrm{~mm}$.

Figure 14. The automated image acquisition and quantification method applied to detecting short range microstructure layer banding in the EBM process; (a) optical macro image showing faint etched layer bands, (b) a SEM high resolution tile map over $~ 3$ layers, (c) the mean $\alpha$ inter lath spacing (ILS) and $\beta$ circularity as a function of height, (d) a Fourier transform of the ILS data plotted as a function of wavelength.

Figure 15 Characterisation of a single bead wide WAAM wall sample, showing quantification of the interlamellar spacing averaged from a 5 tile wide $(125 \mu \mathrm{m})$ stitched image strip, mapped across the last two HAZ layer bands into the fully $\beta$ heat treated region at the top of the wall. The position of the image tile map is shown to scale in the insert optical image. The arrows indicate the positions of the images shown in Figure 8 (a) - (c). 
Table 1. EBM and WAAM build parameters

Table 2. The optimised SEM imaging settings and MAPS settings used for the high-resolution imaging and mapping of the EBM and WAAM microstructures.

\section{Table 1}

\begin{tabular}{lc|ll} 
Arcam A2 machine & & WAAM & \\
\hline Accelerating voltage & $60 \mathrm{kV}$ & Wire feed speed & $1.6\left(\mathrm{~m} \mathrm{~min}^{-1}\right)$ \\
Chamber pressure & $4 \times 10^{-4}-\frac{3}{-}$ & Travel speed & $4.5\left(\mathrm{~mm} \mathrm{~s}^{-1}\right)$ \\
Contouring theme & 4 & Average current & $110(\mathrm{~A})$ \\
infill hatching theme & 19 & Pulse duration & $0.5(\mathrm{~s})$ \\
build layer height & $50 \mu \mathrm{m}$ & Frequency & $10(\mathrm{~Hz})$ \\
working temperature of & $740^{\circ} \mathrm{C}$ & Gas flow rate & $10 \mathrm{~L} \mathrm{~min}^{-1}$ \\
& & Trailing shield gas flow rate & $20 \mathrm{~L} \mathrm{~min}^{-1}$ \\
& & Torch stand-off & $3.5(\mathrm{~mm})$
\end{tabular}

Table 2

\begin{tabular}{ll}
\multicolumn{2}{c}{ FEI Magellan SEM - Image settings } \\
\hline Voltage $(\mathrm{kV})$ & 8.0 \\
Current $(\mathrm{nA})$ & 1.6 \\
& $25(\mathrm{WAAM})$ \\
Field width $(\mu \mathrm{m})$ & $50(\mathrm{EBM})$ \\
Dwell time $(\mathrm{ms})$ & 20
\end{tabular}

